\title{
Stratifications for moduli of sheaves and moduli of quiver representations
}

\author{
Victoria Hoskins
}

\begin{abstract}
We study the relationship between two stratifications on parameter spaces for coherent sheaves and for quiver representations: a stratification by Harder-Narasimhan types and a stratification arising from the geometric invariant theory construction of the associated moduli spaces of semistable objects. For quiver representations, both stratifications coincide, but this is not quite true for sheaves. We explain why the stratifications on various Quot schemes do not coincide and that the correct parameter space to compare such stratifications is the stack of coherent sheaves, where we construct an asymptotic geometric invariant theory stratification and prove that this coincides with the Harder-Narasimhan stratification. Then we relate these stratifications for sheaves and quiver representations using a generalisation of a construction of Álvarez-Cónsul and King.
\end{abstract}

\section{Introduction}

Many moduli spaces can be described as a quotient of a reductive group $G$ acting on a scheme $Y$ using the methods of geometric invariant theory (GIT) developed by Mumford [MFK94]. This depends on a choice of linearisation of the $G$-action which determines an open subscheme $Y^{\text {ss }}$ of $Y$ of semistable points such that the GIT quotient $Y / / G$ is a good quotient of $Y^{\mathrm{ss}}$. In this article, we are interested in two moduli problems with such a GIT construction:

(1) moduli of representations of a quiver $Q$ with relations $\mathcal{R}$,

(2) moduli of coherent sheaves on a polarised projective scheme $\left(X, \mathcal{O}_{X}(1)\right)$.

In the first case, King [Kin94] uses a stability parameter $\theta$ to construct moduli spaces of $\theta$ semistable quiver representations of dimension vector $d$ as a GIT quotient of an affine scheme by a reductive group action linearised by a character $\rho_{\theta}$. In the second case, Simpson [Sim94] constructs a moduli space of semistable sheaves with Hilbert polynomial $P$ as a GIT quotient of a closed subscheme of a Quot scheme by a reductive group action linearised by an ample line bundle.

Received 28 June 2016, accepted in final form 5 June 2017.

2010 Mathematics Subject Classification 14D20, 14L24 (primary), 14D23, 16G20 (secondary).

Keywords: geometric invariant theory, stratifications, moduli of sheaves, moduli of quiver representations.

This journal is (C) Foundation Compositio Mathematica 2018. This article is distributed with Open Access under the terms of the Creative Commons Attribution Non-Commercial License, which permits non-commercial reuse, distribution, and reproduction in any medium, provided that the original work is properly cited. For commercial re-use, please contact the Foundation Compositio Mathematica.

The author was supported by SFB 647 "Space-Time-Matter", project C3 "Algebraic Geometry: Deformations, Moduli and Vector Bundles" and by the Freie Universität Berlin within the Excellence Initiative of the German Research Foundation. 


\section{STRATIFICATIONS IN MODULI PROBLEMS}

For both moduli problems, we compare a Hesselink stratification arising from the GIT construction and a stratification by Harder-Narasimhan (HN) types. An overview of these stratifications is given in Sections 1.2 and 1.3, and we give an outline of our main results in Sections 1.4 and 1.5 .

\subsection{Moduli and stratifications}

One of the most famous uses of stratifications in moduli problems is the Yang-Mills stratification on an infinite-dimensional affine space $\mathcal{A}$ of unitary connections on a $\mathcal{C}^{\infty}$-vector bundle over a smooth complex projective curve $C$ due to Atiyah and Bott [AB83]. The space $\mathcal{A}$ arises in a gauge-theoretic construction of a moduli space of unitary connections, which is diffeomorphic to a moduli space of semistable (algebraic) vector bundles over $C$. This moduli space of connections is constructed as a symplectic reduction using a moment map for the action of a gauge group, and the Yang-Mills stratification is defined by considering the gradient flow of the norm square of this moment map. Remarkably, the Yang-Mills stratification coincides with a stratification by Harder-Narasimhan types of vector bundles, which arises from the notion of stability for vector bundles. Furthermore, these stratifications are used to give formulae for the Betti numbers of the vector bundle moduli space [AB83, Das92].

Bifet, Ghione and Letizia [BGL94] provide an algebraic approach to calculating these Betti numbers: they use elementary modifications of vector bundles over a curve to characterise vector bundles by matrix divisors and construct a HN stratification on an ind-variety of matrix divisors. However, this approach does not generalise to sheaves on higher-dimensional bases, as one cannot use elementary transformations.

A finite-dimensional version of the work of Atiyah and Bott was proved by Kirwan [Kir84] and Ness [Nes84]: for a linear action of a reductive group on a projective variety, the finite stratification associated to the norm square of a moment map agrees with a finite instability stratification arising from GIT (the Hesselink stratification introduced below).

\subsection{Harder-Narasimhan stratifications}

In both of the above moduli problems, there is a notion of semistability for objects that involves verifying an inequality for all sub-objects; typically, this arises from the GIT notion of semistability appearing in the construction of these moduli spaces. The idea of a Harder-Narasimhan (HN) filtration is to construct a unique maximally destabilising filtration for each object in a moduli problem [HN75]. The HN type of an object is a tuple of invariants for the subquotients appearing in its HN filtration.

Every coherent sheaf has a unique HN filtration: for pure sheaves, this result is well known (see [HL97, Theorem 1.3.4]), and the extension to coherent sheaves is due to Rudakov [Rud97]. The HN type of a sheaf is the tuple of Hilbert polynomials of the subquotients in its HN filtration. For quiver representations, there is no canonical notion of HN filtration with respect to the stability parameter $\theta$. Rather, the notion of HN filtration depends on a collection of positive integers $\alpha_{v}$ indexed by the vertices $v$ of the quiver (see Definition 3.4), although often only the choice $\alpha_{v}=1$, for all vertices $v$, is considered. The HN type of a quiver representation is the tuple of dimension vectors of the subquotients in its HN filtration.

For both quiver representations and sheaves, we can stratify the associated moduli stacks (and parameter spaces used in the GIT construction of these moduli spaces) by HN types. 


\section{Hoskins}

\subsection{Hesselink stratifications}

Let $G$ be a reductive group acting on a scheme $Y$, where

(1) $Y$ is affine, $G$ acts linearly and the action is linearised using a character of $G$, or

(2) $Y$ is projective with an ample $G$-linearisation.

Then, by the Hilbert-Mumford criterion [MFK94], it suffices to check GIT semistability on 1parameter subgroups (1-PSs) of $G$. In Section 2, we explain that associated to this linearised action and a choice of norm on the set of conjugacy classes of 1-PSs, there is a Hesselink stratification [Hes79]

$$
Y=\bigsqcup_{\beta \in \mathcal{B}} S_{\beta}
$$

into finitely many disjoint $G$-invariant locally closed subschemes indexed by conjugacy classes of rational 1-PSs of $G$, whose lowest stratum is $Y^{\text {ss }}$. In order to construct this stratification, the idea is to associate to each unstable point a conjugacy class of adapted 1-PSs of $G$, in the sense of Kempf [Kem78], that are most responsible for the instability of this point (this class depends on the choice of norm). This stratification also depends on the choice of linearisation.

These stratifications have a combinatorial nature: the weights of the action of a maximal torus of $G$ determine the index set $\mathcal{B}$, and the strata $S_{\beta}$ can be constructed from simpler limit sets $Z_{\beta}$, which are semistable sets for smaller reductive group actions (cf. Section 2).

Hesselink stratifications have a diverse range of applications. For a smooth projective variety $Y$, the cohomology of $Y / / G$ can be studied via these stratifications [Kir84]. In variation of GIT, these stratifications are used to describe the birational transformations between quotients [DH98, Tha96] and to provide orthogonal decompositions in the derived categories of GIT quotients [BFK17, Hal15]. More recently, Halpern-Leistner abstracted the properties of these instability stratifications in GIT to define instability stratifications on certain classes of "weakly reductive' stacks [Hal14].

\subsection{Comparison results}

As both the HN stratification and the Hesselink stratification are concerned with (in)stability, it is natural to ask whether these stratifications agree. Furthermore, the work of [AB83] suggests that we might expect these stratifications to agree.

For quiver representations, the choice of parameter $\alpha$ used to define HN filtrations corresponds to a choice of norm $\|-\|_{\alpha}$ used to define the Hesselink stratification. The corresponding HN and Hesselink stratifications on the space of quiver representations agree: for a quiver without relations, this result is [Hos14, Theorem 5.5], and we deduce the corresponding result for a quiver with relations in Theorem 3.8.

Simpson constructs the moduli space of (Gieseker) semistable sheaves on $\left(X, \mathcal{O}_{X}(1)\right)$ with Hilbert polynomial $P$ as a GIT quotient of a closed subscheme $R_{n}$ of the Quot scheme

$$
\operatorname{Quot}_{n}:=\operatorname{Quot}\left(k^{P(n)} \otimes \mathcal{O}_{X}(-n), P\right)
$$

by the natural $\mathrm{SL}_{P(n)}$-action for $n$ sufficiently large. The action is linearised by embedding the Quot scheme in a Grassmannian (which depends on choosing $m \gg n$ ). However, the resulting moduli space does not depend on the choice of $m \gg n \gg 0$.

One can consider the Hesselink stratification of Quot $_{n}$ and $R_{n}$ associated to this action and the stratifications by HN types of the quotient sheaves. These stratifications were first compared 


\section{STRATIFICATIONS IN MODULI PROBLEMS}

set-theoretically by the author in joint work with Kirwan $[\mathrm{HK} 12, \S 6]$, where we showed that for a pure HN type $\tau$ (that is, a HN type of a pure sheaf) and for $m \gg n \gg 0$ (depending on $\tau$ ), there is a corresponding Hesselink stratum of $R_{n}$ indexed by $\beta_{n, m}(\tau)$ such that the HN stratum of $R_{n}$ indexed by $\tau$ is contained in this Hesselink stratum. In general, this containment is strict. This result raises the following questions:

(1) Why do we only have a containment of a HN stratum in a Hesselink stratum, rather than an agreement of these stratifications?

(2) As this is an asymptotic result for $m \gg n \gg 0$ depending on $\tau$, is there an asymptotic version of the Hesselink stratifications which agrees with the HN stratification?

The primary aim of this paper is to answer these questions. In this paper, we work with coherent sheaves rather than pure sheaves and study the Hesselink stratifications on Quot ${ }_{n}$. First, we enhance and generalise the result of [HK12] stated above. The proof in op. cit. is settheoretic, whereas we now give a scheme-theoretic proof, and we extend the argument to include non-pure HN types. In Theorem 4.19, we show that for any HN type $\tau$ and $m \gg n \gg 0$, the $\tau$-HN stratum of Quot $_{n}$ is a closed subscheme of a Hesselink stratum indexed by $\beta_{n, m}(\tau)$.

The first question is answered by Lemma 4.22: distinct HN types $\tau$ and $\tau^{\prime}$ can give the same Hesselink index $\beta$ for finitely many values of $n$ and $m$ (although, this only happens when $X$ has dimension greater than 1). Since there are infinitely many HN types, it is not possible to pick finite $n$ and $m$ such that the assignment $\tau \mapsto \beta_{n, m}(\tau)$ is injective. To resolve this issue, we consider a refined version of the Hesselink stratification, where the refined strata $S_{\beta, \nu}$ are further indexed by tuples of polynomials $\nu$ and are (unions of) connected components of the unrefined strata $S_{\beta}$. However, this refined Hesselink stratification still does not agree with the $\mathrm{HN}$ stratification (as not all quotient sheaves parametrised by Quot $_{n}$ are $n$-regular).

The second question is natural, as the Hesselink stratification is a finite stratification, whereas in general there are infinitely many $\mathrm{HN}$ types for sheaves on $X$ with Hilbert polynomial $P$. This suggests that to compare these stratifications properly, one should construct an infinite asymptotic Hesselink stratification. Since every coherent sheaf is $n$-regular for $n$ sufficiently large (depending on the sheaf), one would like to compare the Hesselink stratifications on Quot $_{n}$ for different $n$. This is not as straightforward as in the case of curves studied in [BGL94], as there are no natural maps between these Quot schemes for different values of $n$. However, if we consider the open subscheme $Q^{n \text {-reg }} \subset$ Quot $_{n}$ parametrising $n$-regular quotient sheaves, then there are natural equivariant morphisms $Q^{n \text {-reg }} \rightarrow Q^{n^{\prime} \text {-reg }}$ for $n^{\prime}>n$, as every $n$-regular sheaf is $n^{\prime}$-regular. In fact, the quotient stack of the natural action of $\mathrm{GL}_{P(n)}$ on $Q^{n \text {-reg }}$ is isomorphic to the stack $\mathcal{C}_{\mathrm{oh}}^{n \text {-reg }}(X)$ of $n$-regular coherent sheaves on $X$ with Hilbert polynomial $P$, and the above equivariant morphism of schemes gives an open immersion

$$
\mathcal{C o h}_{P}^{n-\text { reg }}(X) \hookrightarrow \mathcal{C}_{P}^{n^{\prime}-\text { reg }}(X) .
$$

The direct limit of these open immersions is the stack $\mathcal{C} \mathrm{oh}_{P}(X)$ of coherent sheaves on $X$ with

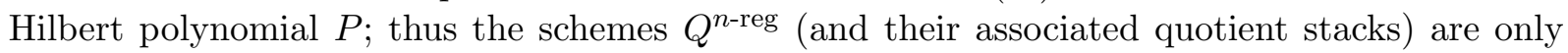
finite-dimensional approximations of $\mathcal{C o h}_{P}(X)$. The natural space on which to construct an asymptotic Hesselink stratification is the stack $\mathcal{C}_{\mathrm{oh}}(X)$.

Let us outline our construction of an asymptotic Hesselink stratification on $\mathcal{C} \mathrm{oh}_{P}(X)$. We restrict the refined Hesselink stratification of Quot $_{n}$ to $Q^{n \text {-reg }}$ and take the stack quotient of each 


\section{Hoskins}

stratum by $\mathrm{GL}_{P(n)}$ to give a (finite) Hesselink stratification

$$
\mathcal{C o h}_{P}^{n-\operatorname{reg}}(X)=\bigsqcup_{(\beta, \nu) \in \mathcal{B}_{n}} \mathcal{S}_{\beta, \nu}^{n}
$$

For a tuple of Hilbert polynomials $\nu$, we define an associated Hesselink index $\beta_{n}(\nu)$ for each $n$ and write $\mathcal{S}_{\nu}^{n}:=\mathcal{S}_{\beta_{n}(\nu), \nu}^{n}$, and, for $n^{\prime}>n$, we construct fibre products

$$
\mathcal{S}_{\nu}^{n, n^{\prime}}:=\mathcal{S}_{\nu}^{n} \times \operatorname{Coh}_{P}^{n^{\prime}-\operatorname{reg}}(X) \mathcal{S}_{\nu}^{n^{\prime}}
$$

which we prove stabilise to an asymptotic stratum denoted by $\mathcal{S}_{\nu}$. Let us state the main result.

Theorem 1.1 (cf. Theorem 4.27). The strata $\mathcal{S}_{\nu}$ determine an asymptotic Hesselink stratification of $\mathcal{C} \mathrm{oh}_{P}(X)$ which coincides with the HN stratification as stacks.

In fact, the asymptotic Hesselink strata $\mathcal{S}_{\nu}$ and the $\mathrm{HN}$ strata $\mathcal{C} \operatorname{oh}_{P}^{\tau}(X)$ can both be realised as global quotient stacks for the same group, and this agreement as stacks comes from a schemetheoretic agreement of the atlases.

The reason why the Hesselink stratification and HN stratifications on the Quot schemes do not agree is that we are only seeing a finite-dimensional approximation of the full picture. One may hope to be able to use this result to study the cohomology ring of the moduli space of semistable sheaves (analogously to [AB83, BGL94]), but there is a major obstruction to such a programme: the subschemes $Q^{n \text {-reg }}$ of the Quot schemes used for curves are all smooth, whereas for higher-dimensional $X$, these Quot schemes can have arbitrarily bad singularities, in which case the results of Kirwan [Kir84] do not apply. Hence different techniques (such as using derived algebraic geometry) are required. Another application of this work is in the construction of moduli spaces of sheaves of a fixed HN type, as initiated in [HK12].

The relationship between filtrations arising from adapted 1-PSs and the Harder-Narasimhan filtration is also studied for torsion-free sheaves on a smooth complex projective variety in [GSZ15] and for quiver representations in [Zam14]; in both cases, an algebraic approach is taken which involves studying convexity properties of a (normalised) Hilbert-Mumford weight.

In this paper and in [Hos14, HK12], we utilise the additional geometric perspective provided by the stratifications and exploit the structure of these stratifications to reduce the proofs to comparisons between simpler limit sets associated to the strata. The use of stratifications enables a direct comparison between the notions of instability coming from GIT and from the HarderNarasimhan filtration, from which we see that these notions do not always coincide (as is the case for the Quot scheme). A key motivation for this work was to explain why this happens. Furthermore, stratifications can be used to study GIT situations where 1-PSs do not give rise to filtrations by sub-objects (for example, in the study of moduli of curves, there is no natural notion of sub-object).

\subsection{The functorial construction of Álvarez-Cónsul and King}

In Section 5, we relate these stratifications for sheaves and quivers using a generalisation of [AK07]. There, a functor

$$
\Phi_{n, m}:=\operatorname{Hom}\left(\mathcal{O}_{X}(-n) \oplus \mathcal{O}_{X}(-m),-\right): \operatorname{Coh}(X) \rightarrow \operatorname{Rep}\left(K_{n, m}\right)
$$

from the category of coherent sheaves on $X$ to the category of representations of a Kronecker quiver $K_{n, m}$ with two vertices $n, m$ and $\operatorname{dim} H^{0}\left(\mathcal{O}_{X}(m-n)\right.$ ) arrows from $n$ to $m$ is used (when $m \gg n \gg 0)$ to embed the subcategory of semistable sheaves with Hilbert polynomial $P$ into 


\section{STRATIFICATIONS IN MODULI PROBLEMS}

a subcategory of $\theta_{n, m}(P)$-semistable quiver representations of dimension $d_{n, m}(P)$ and to construct the moduli space of semistable sheaves on $X$ with Hilbert polynomial $P$ using [Kin94].

In this setup, distinct HN strata for sheaves may be sent via $\Phi_{n, m}$ to the same HN stratum for quiver representations. We resolve this problem by generalising the above construction to allow additional vertices, as follows (cf. Remark 5.16). For a tuple of strictly increasing positive integers $\underline{n}=\left(n_{0}, \ldots, n_{d}\right)$, we consider a functor

$$
\Phi_{\underline{n}}:=\operatorname{Hom}\left(\bigoplus_{i=0}^{d} \mathcal{O}_{X}\left(-n_{i}\right),-\right): \operatorname{Coh}(X) \rightarrow \boldsymbol{\operatorname { R e p }}\left(K_{\underline{n}}\right),
$$

where $K_{n}$ is a quiver with $d+1$ vertices and $\operatorname{dim} H^{0}\left(\mathcal{O}_{X}\left(n_{i+1}-n_{i}\right)\right)$ arrows from vertex $i$ to vertex $i+1$ and relations as described in Section 5.4. We provide stability parameters $\left(\theta_{\underline{n}}(P), \alpha_{\underline{n}}(P)\right)$ such that a HN stratum for sheaves is mapped via $\Phi_{\underline{n}}$ to a HN stratum for quiver representations, provided that the tuple of natural numbers $\underline{n}$ are sufficiently large (see Theorem 5.15 ).

\section{Notation and conventions}

We work over an algebraically closed field $k$ of characteristic zero, and by scheme, we mean scheme of finite type over $k$. By sheaf, we always mean coherent algebraic sheaf. For schemes $X$ and $S$, by a family of sheaves on $X$ parametrised by $S$, we mean a sheaf $\mathcal{F}$ over $X \times S$ that is flat over $S$, and we write $\mathcal{F}_{s}:=\left.\mathcal{F}\right|_{X \times\{s\}}$.

We use the term stratification in a weaker sense than usual to mean a decomposition into disjoint locally closed subschemes with a partial order on the strata such that the closure of a given stratum is contained in the union of all higher strata (usually for a stratification, one requires the closure of a given stratum to be the union of all higher strata).

For $m, n \in \mathbb{N}$, we write 'for $m \gg n$ ' to mean that there exists an $N \geqslant n$ such that for all $m \geqslant N$. Similarly, 'for $n_{r} \gg \cdots \gg n_{0}$ ' means $\exists N_{0} \geqslant n_{0} \forall n_{1} \geqslant N_{0} \ldots \exists N_{r-1} \geqslant n_{r-1}$ $\forall n_{r} \geqslant N_{r-1}$.

\section{Background on GIT and Hesselink stratifications}

Let $G$ be a reductive group acting on a scheme $Y$ with respect to a linearisation $L$ over $Y$. Throughout this section, we assume that we are in one of the two following settings:

(1) The scheme $Y \subset \mathbb{A}^{n}$ is affine, the group $G$ acts linearly on $Y$, and the linearisation $L$ is given by using a character $\rho: G \rightarrow \mathbb{G}_{m}$ to twist the trivial linearisation on the structure sheaf of $Y$,

(2) The scheme $Y$ is projective and the linearisation $L$ is ample.

There are two reasons for working in such settings: (1) in both these settings, there is a HilbertMumford criterion, as explained below, and (2) these two settings are used to give the GIT constructions of the two moduli spaces considered in this paper.

\subsection{GIT and the construction of Hesselink stratifications}

Definition 2.1. A point $y \in Y$ is $L$-semistable if there exists a $\sigma \in H^{0}(Y, L)^{G}$ for some $n>0$ such that $\sigma(y) \neq 0$. We let $Y^{\mathrm{ss}}(L)$ denote the subset of $L$-semistable points in $Y$. 


\section{Hoskins}

By [MFK94, Theorem 1.10], the GIT quotient of $G$ acting on $Y$ with respect to $L$,

$$
Y^{\mathrm{ss}}(L) \rightarrow Y / /{ }_{L} G:=\operatorname{Proj}\left(\oplus_{n \geqslant 0} H^{0}\left(Y, L^{\otimes n}\right)^{G}\right),
$$

is a good quotient. In Setting (2), this quotient is projective and in Setting (1), this quotient is projective over the affine GIT quotient $Y / / G:=\operatorname{Spec} k[Y]^{G}$.

Definition 2.2. Let $y \in Y$ be a closed point and $\lambda: \mathbb{G}_{m} \rightarrow G$ be a 1-parameter subgroup (1-PS) of $G$; then the Hilbert-Mumford weight $\mu^{L}(y, \lambda) \in \mathbb{Z}$ is defined to be the weight of the $\mathbb{G}_{m}$-action induced by $\lambda$ on the fibre of $L$ over the fixed point $\lim _{t \rightarrow 0} \lambda(t) \cdot y$, if this limit exists.

The limit is unique, as in both settings $Y$ is separated and, in Setting (2), the limit always exists as $Y$ is proper. In Setting (1), by using the pairing $(-,-): X^{*}(G) \times X_{*}(G) \rightarrow \mathbb{Z}$ between characters and cocharacters, King [Kin94] shows that $\mu^{L}(y, \lambda)=(\rho, \lambda)$.

TheOrem 2.3 (Hilbert-Mumford criterion [Kin94, MFK94]). A closed point $y \in Y$ is $L$-semistable if and only we have $\mu^{L}(y, \lambda) \geqslant 0$ for every $1-P S \lambda: \mathbb{G}_{m} \rightarrow G$ such that $\lim _{t \rightarrow 0} \lambda(t) \cdot y$ exists.

The unstable locus $Y^{\mathrm{us}}(L):=Y-Y^{\mathrm{ss}}(L)$ can be stratified by instability types by assigning to each unstable point a conjugacy class of (rational) 1-PSs that is 'most responsible' for the instability of this point [Kem78, Hes79]. Here, the term 'most responsible' is measured using a norm $\|-\|$ on the set $\bar{X}_{*}(G)$ of conjugacy classes of 1-PSs of $G$. More precisely, we fix a maximal torus $T$ of $G$ and a Weyl-invariant norm $\|-\|_{T}$ on $X_{*}(T)_{\mathbb{R}}$; then, for $\lambda \in X_{*}(G)$, we let $\|\lambda\|:=\left\|g \lambda g^{-1}\right\|_{T}$ for $g \in G$ such that $g \lambda g^{-1} \in X_{*}(T)$. Equivalently, the norm is given by a Weyl-invariant inner product on the Lie algebra of $T$.

Example 2.4. (a) Let $T$ be the diagonal maximal torus in $G=\mathrm{GL}_{n}$; then the norm associated to the dot product on $\mathbb{R}^{n} \cong X_{*}(T)_{\mathbb{R}}$ is invariant under the Weyl group $W=S_{n}$.

(b) For a product of general linear groups $G=\mathrm{GL}_{n_{1}} \times \cdots \times \mathrm{GL}_{n_{r}}$, we can use positive numbers $\alpha \in \mathbb{N}^{r}$ to weight the norms $\|-\|$ for each factor constructed by part (a); that is,

$$
\left\|\left(\lambda_{1}, \ldots, \lambda_{r}\right)\right\|_{\alpha}^{2}:=\sum_{i=1}^{r} \alpha_{i}\left\|\lambda_{i}\right\|^{2} .
$$

We fix a norm $\|-\|$ on $\bar{X}_{*}(G)$ such that $\|-\|^{2}$ is $\mathbb{Z}$-valued. For a closed point $y \in Y$, we let

$$
M^{L}(y):=\inf \left\{\frac{\mu^{L}(y, \lambda)}{\|\lambda\|}: \lambda \text { is a 1-PS of } G \text { such that } \lim _{t \rightarrow 0} \lambda(t) \cdot y \text { exists }\right\} .
$$

By the Hilbert-Mumford criterion, $y$ is $L$-semistable if and only if $M^{L}(y) \geqslant 0$.

Definition 2.5 (Kempf). A 1-PS $\lambda$ is adapted to $y \in Y^{\mathrm{us}}(L)(k)$ if $\lim _{t \rightarrow 0} \lambda(t) \cdot y$ exists and

$$
M^{L}(y)=\frac{\mu^{L}(y, \lambda)}{\|\lambda\|}
$$

Let $\wedge^{L}(y)$ denote the set of primitive 1-PSs which are adapted to $y$; this is a full conjugacy class of 1-PSs for a parabolic subgroup of $G$ [Kem78]. Kempf proves that $\wedge^{L}(g \cdot y)=g \wedge^{L}(y) g^{-1}$ for $g \in G$; thus, in order to have $G$-invariant strata, we need to stratify the unstable locus by conjugacy classes of primitive 1-PSs (and an additional index $d$ which keeps track of the value of $M^{L}$ ). We first define the $k$-points of the strata; the scheme structure is given in Remark 2.9. 


\section{STRATIFICATIONS IN MODULI PROBLEMS}

Definition 2.6 (Hesselink [Hes79]; Kirwan [Kir84] and Ness [Nes84]). For a conjugacy class $[\lambda]$ of 1-PSs of $G$, a choice of representative $\lambda \in[\lambda]$ and $d \in \mathbb{Q}>0$, we define the following subsets of $Y(k)$ :

$$
\begin{array}{lll}
\text { (i) } & S_{[\lambda], d}:=\left\{y \in Y(k): M^{L}(y)=-d \text { and } \wedge^{L}(y) \cap[\lambda] \neq \emptyset\right\} & \text { 'the strata', } \\
\text { (ii) } S_{d}^{\lambda}=\left\{y \in Y(k): M^{L}(y)=-d \text { and } \lambda \in \wedge^{L}(y)\right\} & \text { 'the blades', } \\
\text { (iii) } Z_{d}^{\lambda}=\left\{y \in Y^{\lambda}(k): M^{L}(y)=-d \text { and } \lambda \in \wedge^{L}(y)\right\} & \text { 'the limit sets', }
\end{array}
$$

where $Y^{\lambda}$ denotes the $\lambda$-fixed locus in $Y$.

Remark 2.7. The additional index $d$ is redundant in the affine Setting (1), as $d$ can be determined from the character $\rho$ and 1-PS $\lambda$ (more precisely, we have $d=-(\rho, \lambda) /\|\lambda\|)$.

Let $Y_{+}^{\lambda} \subset Y$ denoted the closed subscheme of points $y \in Y$ such that $\lim _{t \rightarrow 0} \lambda(t) \cdot y$ exists; then there is a natural retraction $p_{\lambda}: Y_{+}^{\lambda} \rightarrow Y^{\lambda}$. Let $Y_{d}^{\lambda} \subset Y^{\lambda}$ be the union of connected components on which $\mu^{L}(-, \lambda)=-d\|\lambda\|$. Let $G_{\lambda}$ be the commutator of $\lambda$ in $G$ and let $P(\lambda):=\{g \in G$ : $\lim _{t \rightarrow 0} \lambda(t) g \lambda(t)^{-1}$ exists $\}$.

Theorem 2.8 ([Hes79, Kir84, Nes84]). Let $G$ be a reductive group acting on a scheme $Y$ with respect to a linearisation $L$, as in either Setting (1) or (2) above, and let $\|$ - $\|$ be a norm on conjugacy classes of 1-PSs of $G$. Then there is a stratification

$$
Y-Y^{\mathrm{ss}}(L)=\bigsqcup_{([\lambda], d)} S_{[\lambda], d}
$$

into finitely many disjoint $G$-invariant locally closed subschemes $S_{[\lambda], d}$ of $Y$. Moreover,

(i) $S_{[\lambda], d}=G \cdot S_{d}^{\lambda}$;

(ii) $S_{d}^{\lambda}=p_{\lambda}^{-1}\left(Z_{d}^{\lambda}\right)$ and $S_{d}^{\lambda}$ is $P(\lambda)$-invariant;

(iii) $Z_{d}^{\lambda}$ is $G_{\lambda}$-invariant and is the GIT semistable locus for $G_{\lambda}$ acting on $Y_{d}^{\lambda}$ with respect to a modified linearisation;

(iv) $\partial S_{[\lambda], d} \cap S_{\left[\lambda^{\prime}\right], d^{\prime}} \neq \emptyset$ only if $d>d^{\prime}$.

Remark 2.9. This theorem was proved in [Hes79, Kir84, Nes84] for a projective variety $Y$, where the strata are shown to be locally closed subvarieties of $Y$. However, as observed in [HK12, $\S 4]$, this can be extended to the case where $Y$ is a scheme by using the ample linearisation $L$ on $Y$ to give a projective embedding $Y \hookrightarrow \mathbb{P}^{N}$; then we obtain the strata in $Y$ by taking the fibre product of the strata in $\mathbb{P}^{N}$ with $Y$ (and similarly for the blades and limit sets). This gives each stratum the structure of a locally closed subscheme of $Y$.

This theorem was proved in [Hos14] for an affine space $Y$; however, the result for an affine subscheme $Y \subset \mathbb{A}^{n}$ with a linear $G$-action can be deduced from this, by using the fact that taking invariants for a reductive group $G$ is exact; thus $Y^{\mathrm{ss}}(L)=Y \times_{\mathbb{A}^{n}}\left(\mathbb{A}^{n}\right)^{\mathrm{ss}}(L)$, and, similarly, the Hesselink strata in $Y$ are obtained as fibre products of the Hesselink strata in $\mathbb{A}^{n}$.

By setting $S_{0}:=Y^{\mathrm{ss}}(L)$, we obtain the Hesselink stratification of $Y$

$$
Y=\bigsqcup_{\beta} S_{\beta},
$$

where the lowest stratum is $S_{0}$ and the higher strata are indexed by pairs $\beta=([\lambda], d)$ as above or equivalently by a conjugacy class of a rational 1-PS $\left[\lambda_{\beta}\right]$, by the following remark. 


\section{Hoskins}

Remark 2.10. An unstable Hesselink index $\beta=([\lambda], d)$ determines a rational 1-PS

$$
\lambda_{\beta}:=\frac{d}{\|\lambda\|} \lambda
$$

(where $d /\|\lambda\|$ is rational, as $\|\lambda\|^{2}$ and $d\|\lambda\|$ are both integral). In fact, we can recover $([\lambda], d)$ from $\lambda_{\beta}$, as $d=-\left\|\lambda_{\beta}\right\|$ and $\lambda$ is the unique primitive 1-PS lying on the ray spanned by $\lambda_{\beta}$.

Hesselink stratifications have the following additional properties:

(1) For a closed $G$-invariant subscheme $Y^{\prime}$ of $Y$, the Hesselink strata in $Y^{\prime}$ are the fibre products of the Hesselink strata in $Y$ with $Y^{\prime}$.

(2) The Hesselink strata may not be connected; however, if we let $Z_{d, i}^{\lambda}$ denote the connected components of $Z_{d}^{\lambda}$, then the $S_{[\lambda], d, i}:=G p_{\lambda}^{-1}\left(Z_{d, i}^{\lambda}\right)$ are the connected components of $S_{[\lambda], d}$.

(3) If $\left(Z_{d}^{\lambda}\right)^{\prime}$ is a closed subscheme of $Z_{d}^{\lambda}$, then there is a naturally associated closed $G$-invariant subscheme $S_{[\lambda], d}^{\prime}$ of $S_{[\lambda], d}$ such that $S_{[\lambda], d}^{\prime}=G \cdot p_{\lambda}^{-1}\left(\left(Z_{d}^{\lambda}\right)^{\prime}\right)$.

\subsection{Computing Hesselink stratifications}

The task of computing these stratifications is greatly simplified by Theorem 2.8, as we can construct the strata from the limit sets $Z_{\lambda}^{d}$. Furthermore, we will see that the index set $\mathcal{B}$ for the stratification can be determined from the weights of a maximal torus $T$ of $G$.

In the projective Setting (2), this description is due to Kirwan [Kir84]: for $G$ acting on $\mathbb{P}^{n}$ linearly via a representation $\varphi: G \rightarrow \mathrm{GL}_{n+1}$, the indices can be determined from the weights of $T$ as follows. For each subset of the $T$-weights, let $\beta$ denote the closest point to the origin in the convex hull of these weights. Then, for $\beta \neq 0$, there is a unique primitive 1-PS $\lambda$ lying on the ray through $\beta$, and we let $d:=\|\beta\|$; then $\beta=([\lambda], d)$ is an unstable index.

In the affine Setting (1), we obtain a similar description as follows. First, we recall that in the affine setting, the index $d$ is redundant and, as the indices for $Y \subset V:=\mathbb{A}^{n}$ are obtained from the indices for $V$, it suffices to consider the case of an affine space $V=\mathbb{A}^{n}$ with a linear action $G \rightarrow \mathrm{GL}_{n}$. For each subset $W$ of $T$-weights, we consider an associated cone

$$
C_{W}:=\bigcap_{\chi \in W} H_{\chi} \subset X_{*}(T)_{\mathbb{R}}, \quad \text { where } H_{\chi}:=\left\{\lambda \in X_{*}(T)_{\mathbb{R}}:(\lambda, \chi) \geqslant 0\right\} .
$$

Let $\rho_{T} \in X^{*}(T)$ denote the restriction of $\rho$ to $T$; then a subset $W$ of the $T$-weights is called $\rho_{T}$-semistable if $C_{W} \subseteq H_{\rho_{T}}$, and otherwise we say that $W$ is $\rho_{T}$-unstable. If $W$ is $\rho_{T}$-unstable, we let $\lambda_{W}$ be the unique primitive 1-PS in $C_{W} \cap X_{*}(T)$ for which $(\lambda, \rho) /\|\lambda\|$ is minimal (for the existence and uniqueness of this 1-PS, see [Hos14, Lemma 2.13]). We will shortly prove that this gives an unstable index. For $v \in V$, we let $W_{v}$ denote the set of $T$-weights of $v$.

Proposition 2.11. Let $T=\left(\mathbb{G}_{m}\right)^{n}$ act linearly on an affine space $V$ with respect to a character $\rho: T \rightarrow \mathbb{G}_{m}$, and let $\|-\|$ be the norm associated to the dot product on $\mathbb{Z}^{n}$; then the Hesselink stratification for the torus $T$ is given by

$$
V-V^{\rho \text {-ss }}=\bigsqcup_{\{W: W \text { is } \rho \text {-unstable }\}} S_{\lambda_{W}}, \quad \text { where } S_{\lambda_{W}}=\left\{v \in V: W=W_{v}\right\} .
$$

Therefore, the stratification is determined by the $T$-weights, and, moreover, $v \in V$ is $\rho$-semistable if and only if its $T$-weight set $W_{v}$ is $\rho$-semistable (that is, $C_{W_{v}} \subseteq H_{\rho}$ ).

Proof. By construction, $C_{W_{v}}$ is the cone of (real) 1-PSs $\lambda$ such that $\lim _{t \rightarrow 0} \lambda(t) \cdot v$ exists. By the 


\section{STRATIFICATIONS IN MODULI PROBLEMS}

Hilbert-Mumford criterion, $v$ is $\rho$-semistable if and only if $(\lambda, \rho) \geqslant 0$ for all $\lambda \in C_{W_{v}}$, that is, if and only if $C_{W_{v}} \subseteq H_{\rho}$. Therefore, $v$ is $\rho$-semistable if and only if $W_{v}$ is $\rho$-semistable.

As the conjugation action is trivial, $\left[\lambda_{W}\right]=\lambda_{W}$ and every $\rho$-unstable point has a unique $\rho$-adapted primitive 1-PS. If $v$ is $\rho$-unstable, then, by definition, $\lambda_{W_{v}}$ is $\rho$-adapted to $v$.

For a reductive group $G$, we fix a maximal torus $T$ of $G$ and positive Weyl chamber. Let $\mathcal{B}$ denote the set of 1-PSs $\lambda_{W}$ corresponding to elements in this positive Weyl chamber where $W$ is a $\rho_{T}$-unstable set of weights.

Corollary 2.12. Let $G$ be a reductive group acting linearly on an affine variety $V$ with respect to a character $\rho: G \rightarrow \mathbb{G}_{m}$. Then the Hesselink stratification is given by

$$
V-V^{\rho-s s}=\bigsqcup_{\lambda_{W} \in \mathcal{B}} S_{\left[\lambda_{W}\right]} .
$$

Proof. It is clear that the left-hand side is contained in the right-hand side. Conversely, suppose that $v \in V-V^{\rho \text {-ss }}$ and $\lambda$ is a primitive 1-PS of $G$ that is $\rho$-adapted to $v$. Then there exists a $g \in G$ such that $\lambda^{\prime}:=g \lambda g^{-1} \in X_{*}(T)$ and, moreover, $\lambda^{\prime}$ is $\rho$-adapted to $v^{\prime}:=g \cdot v$ for the $G$-action on $V$. As every 1-PSs of $T$ is a 1-PSs of $G$, it follows that $M_{G}^{\rho}\left(v^{\prime}\right) \leqslant M_{T}^{\rho_{T}}\left(v^{\prime}\right)$. Therefore, $\lambda^{\prime}$ is $\rho_{T}$-adapted to $v^{\prime}$ for the $T$-action on $V$ and $\lambda^{\prime}=\lambda_{W} \in \mathcal{B}$, where $W=W_{v^{\prime}}$. In particular, we have that $v \in S_{[\lambda]}=S_{\left[\lambda_{W}\right]}$.

This gives an algorithm to compute the Hesselink stratification: the index set $\mathcal{B}$ is determined from the $T$-weights and, for each $[\lambda] \in \mathcal{B}$, we construct $S_{[\lambda]}$ from $Z_{\lambda}$ using Theorem 2.8.

Remark 2.13. In general, the Hesselink stratification for $G$ and its maximal torus $T$ are not easily comparable. The $\rho$-semistable locus for the $G$-action is contained in the $\rho$-semistable locus for the $T$-action, but the $G$-stratification does not refine the $T$-stratification. Often there are more $T$-strata, since 1-PSs of $T$ may be conjugate in $G$ but not in $T$.

Example 2.14. Let $G:=\mathrm{GL}_{r}$ act on $V:=\mathrm{Mat}_{r \times n} \cong \mathbb{A}^{r n}$ by left multiplication; then

$$
\text { Mat }_{r \times n} / / \operatorname{det} \mathrm{GL}_{r}=\operatorname{Gr}(r, n)
$$

is the Grassmannian of $r$-planes in $\mathbb{A}^{n}$, and the GIT semistable locus is given by matrices of rank $r$. We claim that the Hesselink stratification is the stratification given by rank.

We fix the maximal torus $T \cong \mathbb{G}_{m}^{r}$ of diagonal matrices in $G$ and use the dot product on $\mathbb{Z}^{r} \cong X_{*}(T)$ to define our norm $\|-\|$. The weights of the $T$-action on $V$ are $\chi_{1}, \ldots, \chi_{r}$, where $\chi_{i}$ denotes the $i$ th standard basis vector in $\mathbb{Z}^{r}$ and the Weyl group $S_{r}$ acts transitively on the $T$-weights. The restriction of the determinant to $T$ is given by $\operatorname{det}_{T}=(1, \ldots, 1) \in \mathbb{Z}^{r} \cong X^{*}(T)$. As the Weyl group is the full permutation group on the set of $T$-weights, it follows that for $\operatorname{det}_{T}$-unstable weight subsets $W$ and $W^{\prime}$, the corresponding 1-PSs $\lambda_{W}$ and $\lambda_{W^{\prime}}$ are conjugate under $S_{r}$ whenever $|W|=\left|W^{\prime}\right|$. Thus, it suffices to consider the weight sets

$$
W_{k}:=\left\{\chi_{1}, \ldots, \chi_{k}\right\} \quad \text { for } k=0, \ldots, r .
$$

The subset $W_{k}$ is $\rho_{T}$-unstable if and only if $k<r$ with corresponding $\rho_{T}$-adapted 1-PS

$$
\lambda_{k}:=(\underbrace{0, \ldots, 0}_{k}, \underbrace{-1, \ldots,-1}_{r-k}) .
$$

By Corollary 2.12, the index set for the stratification is $\mathcal{B}=\left\{\lambda_{k}: 0 \leqslant k \leqslant r-1\right\}$. We use Theorem 2.8 to calculate the unstable strata. If we consider Mat $\operatorname{Man}_{k \times n}$ as the subvariety of Mat $r \times n$ 


\section{Hoskins}

consisting of matrices whose last $r-k$ rows are zero, then $V_{+}^{\lambda_{k}}=V^{\lambda_{k}}=$ Mat $_{k \times n}$ and thus $S_{\lambda_{k}}=Z_{\lambda_{k}}$. By definition, $Z_{\lambda_{k}}$ is the GIT semistable set for $G_{\lambda_{k}} \cong \mathrm{GL}_{k} \times \mathrm{GL}_{r-k}$ acting on $V^{\lambda_{k}}$ with respect to the character

$$
\operatorname{det}_{\lambda_{k}}:=\left\|\lambda_{k}\right\|^{2} \rho-\left(\lambda_{k}, \rho\right) \lambda_{k}=(r-k) \operatorname{det}-(k-r) \lambda_{k}=(\underbrace{r-k, \ldots, r-k}_{k}, \underbrace{0, \ldots, 0}_{r-k}) .
$$

As semistability is unchanged by a positive rescaling of the character, we can without loss of generality assume that $\rho_{\lambda_{k}}$ is the product of the determinant character on $\mathrm{GL}_{k}$ and the trivial character on $\mathrm{GL}_{r-k}$. Then $Z_{\lambda_{k}}$ is the GIT semistable set for $\mathrm{GL}_{k}$ acting on $\mathrm{Mat}_{k \times n} \cong V^{\lambda_{k}}$ with respect to det: $\mathrm{GL}_{k} \rightarrow \mathbb{G}_{m}$; that is, $Z_{\lambda_{k}}$ is the set of matrices in Mat $k \times n$ whose final $r-k$ rows are zero and whose top $k$ row vectors are linearly independent. Then, $S_{\left[\lambda_{k}\right]}=G \cdot S_{\lambda_{k}}$ is the locally closed subvariety of rank $k$ matrices, and the Hesselink stratification is given by rank.

\section{Stratifications for moduli of quiver representations}

Let $Q=(V, A, h, t)$ be a quiver with vertex set $V$, arrow set $A$ and head and tail maps $h, t: A \rightarrow V$ giving the direction of the arrows. A $k$-representation of a quiver $Q=(V, A, h, t)$ is a tuple $W=$ $\left(W_{v}, \phi_{a}\right)$ consisting of a $k$-vector space $W_{v}$ for each vertex $v$ and a linear map $\phi_{a}: W_{t(a)} \rightarrow W_{h(a)}$ for each arrow $a$. The dimension vector of $W$ is $\operatorname{dim} W=\left(\operatorname{dim} W_{v}\right) \in \mathbb{N}^{V}$.

\subsection{Semistable quiver representations}

For quiver representations of a fixed dimension vector $d=\left(d_{v}\right) \in \mathbb{N}^{V}$, King introduced a notion of semistability depending on a stability parameter $\theta=\left(\theta_{v}\right) \in \mathbb{Z}^{V}$ such that $\sum_{v \in V} \theta_{v} d_{v}=0$.

Definition 3.1. A representation $W$ of $Q$ of dimension $d$ is $\theta$-semistable if for all subrepresentations $W^{\prime} \subset W$, we have $\theta\left(W^{\prime}\right):=\sum_{v} \theta_{v} \operatorname{dim} W_{v}^{\prime} \geqslant 0$.

We recall King's construction of moduli of $\theta$-semistable quiver representations [Kin94]. Let

$$
\operatorname{Rep}_{d}(Q):=\bigoplus_{a \in A} \operatorname{Hom}\left(k^{d_{t(a)}}, k^{d_{h(a)}}\right) \quad \text { and } \quad G_{d}(Q):=\prod_{v \in V} \mathrm{GL}_{d_{v}} .
$$

Then $G_{d}(Q)$ acts on $\operatorname{Rep}_{d}(Q)$ by conjugation. Since the diagonal subgroup $\mathbb{G}_{m} \cong \Delta \subset G_{d}(Q)$, given by $t \in \mathbb{G}_{m} \mapsto\left(t I_{d_{v}}\right)_{v \in V} \in G_{d}(Q)$, acts trivially on $\operatorname{Rep}_{d}(Q)$, we often consider the quotient group $\bar{G}_{d}(Q):=G_{d}(Q) / \Delta$. We note that every representation of $Q$ of dimension $d$ is isomorphic to an element of $\operatorname{Rep}_{d}(Q)$ and, moreover, two representations in $\operatorname{Rep}_{d}(Q)$ are isomorphic if and only if they lie in the same $\bar{G}_{d}(Q)$-orbit. Hence, the moduli stack $\mathcal{R e p}_{d}(Q)$ of representations of $Q$ of dimension $d$ is isomorphic to the quotient stack of $\bar{G}_{d}(Q)$ acting on $\operatorname{Rep}(Q)$. The $G_{d}(Q)$-action on $\operatorname{Rep}_{d}(Q)$ is linearised by $\rho_{\theta}: G_{d}(Q) \rightarrow \mathbb{G}_{m}$, where $\rho_{\theta}\left(g_{v}\right):=\Pi_{v} \operatorname{det}\left(g_{v}\right)^{\theta_{v}}$.

THEOREM 3.2 (King [Kin94]). The moduli space of $\theta$-semistable quiver representations is given by $M_{d}^{\theta-\mathrm{ss}}(Q):=\operatorname{Rep}_{d}(Q) / / \rho_{\theta} G_{d}(Q)$.

In particular, King shows that the notion of $\theta$-semistability for quiver representations agrees with the notion of GIT semistability for $G_{d}(Q)$ acting on $\operatorname{Rep}_{d}(Q)$ with respect to $\rho_{\theta}$.

Remark 3.3. We can also consider moduli spaces of $\theta$-semistable quiver representations for quivers with relations. A non-trivial path in a quiver is a sequence of arrows in the quiver $\left(a_{1}, \ldots, a_{n}\right)$ such that $h\left(a_{i}\right)=t\left(a_{i+1}\right)$, and a relation is a linear combination of paths all of which start at some common vertex $v_{t}$ and end at a common vertex $v_{h}$. Let $\mathcal{R}$ be a set of relations on $Q$, and 


\section{STRATIFICATIONS IN MODULI PROBLEMS}

let $\operatorname{Rep}_{d}(Q, \mathcal{R})$ be the closed subscheme of $\operatorname{Rep}_{d}(Q)$ consisting of quiver representations that satisfy the relations $\mathcal{R}$. The moduli space of $\rho$-semistable representations of $(Q, \mathcal{R})$ is a closed subscheme of the moduli space of $\rho$-semistable representations of $Q$ :

$$
M_{d}^{\theta-\mathrm{ss}}(Q, \mathcal{R}):=\operatorname{Rep}_{d}(Q, \mathcal{R}) / / \rho_{\theta} G_{d}(Q) \subset M_{d}^{\theta-\mathrm{ss}}(Q) .
$$

\subsection{HN filtrations for quivers representations}

We fix a quiver $Q$, a dimension vector $d \in \mathbb{N}^{V}$ and a stability parameter $\theta=\left(\theta_{v}\right) \in \mathbb{Z}^{V}$ such that $\sum_{v} \theta_{v} d_{v}=0$. To define a Harder-Narasimhan filtration, we need a notion of semistability for quiver representations of all dimension vectors, and for this we use a parameter $\alpha=\left(\alpha_{v}\right) \in \mathbb{N}^{V}$.

Definition 3.4. A representation $W$ of $Q$ is $(\theta, \alpha)$-semistable if for all $0 \neq W^{\prime} \subset W$, we have

$$
\frac{\theta\left(W^{\prime}\right)}{\alpha\left(W^{\prime}\right)} \geqslant \frac{\theta(W)}{\alpha(W)}
$$

where $\alpha(W):=\sum_{v \in V} \alpha_{v} \operatorname{dim} W_{v}$. A Harder-Narasimhan (HN) filtration of $W$ (with respect to $\theta$ and $\alpha$ ) is a filtration $0=W^{(0)} \subset W^{(1)} \subset \cdots \subset W^{(s)}=W$ by subrepresentations, such that the quotient representations $W_{i}:=W^{(i)} / W^{(i-1)}$ are $(\theta, \alpha)$-semistable and

$$
\frac{\theta\left(W_{1}\right)}{\alpha\left(W_{1}\right)}<\frac{\theta\left(W_{2}\right)}{\alpha\left(W_{2}\right)}<\cdots<\frac{\theta\left(W_{s}\right)}{\alpha\left(W_{s}\right)} .
$$

The Harder-Narasimhan type of $W$ (with respect to $\theta$ and $\alpha)$ is $\gamma(W):=\left(\operatorname{dim} W_{1}, \ldots, \operatorname{dim} W_{s}\right)$.

If $W$ is a representation of dimension $d$, then $\theta(W)=0$ and so $W$ is $(\theta, \alpha)$-semistable if and only if it is $\theta$-semistable. Using the existence and uniqueness of the HN filtration with respect to $(\theta, \alpha)$, Reineke [Rei03] proves that there is a HN stratification (with respect to $\theta$ and $\alpha$ )

$$
\operatorname{Rep}_{d}(Q)=\bigsqcup_{\gamma} R_{\gamma}
$$

where $R_{\gamma}$ is the locally closed subvariety consisting of representations with HN type $\gamma$. As isomorphic quiver representations have the same $\mathrm{HN}$ type, each $\mathrm{HN}$ stratum $R_{\gamma}$ is invariant under the action of $\bar{G}_{d}(Q)$, and so we obtain a HN stratification of the moduli stack

$$
\mathcal{R e p}_{d}(Q)=\bigsqcup_{\gamma} \operatorname{Rep}_{d}^{\gamma}(Q), \quad \text { where } \operatorname{Rep}_{d}^{\gamma}(Q) \cong\left[R_{\gamma} / \bar{G}_{d}(Q)\right]
$$

We emphasise that this stratification depends on both $\theta$ and $\alpha$.

Remark 3.5. For a quiver with relations $(Q, \mathcal{R})$, there is also a HN stratification of the representation space $\operatorname{Rep}_{d}(Q, \mathcal{R})$. If $W$ is a representation of $Q$ that satisfies the relations $\mathcal{R}$, then any subrepresentation of $W$ also satisfies these relations. Therefore, the HN strata of $\operatorname{Rep}_{d}(Q, \mathcal{R})$ are the intersections of the HN strata in $\operatorname{Rep}_{d}(Q)$ with $\operatorname{Rep}_{d}(Q, \mathcal{R})$.

\subsection{The HN stratification is the Hesselink stratification}

Let $T$ be the maximal torus of $G_{d}(Q)$ given by the product of the maximal tori $T_{v} \subset \mathrm{GL}_{d_{v}}$ of diagonal matrices.

DeFinition 3.6. For a HN type $\gamma=\left(d_{1}, \ldots, d_{s}\right)$ of a quiver representation of dimension $d$, let $\lambda_{\gamma}$ be the unique primitive 1-PS on the ray through the rational 1-PS $\lambda_{\gamma}^{\prime}=\left(\lambda_{\gamma, v}^{\prime}\right)$ given by

$$
\lambda_{\gamma, v}^{\prime}(t)=\operatorname{diag}\left(t^{r_{1}}, \ldots, t^{r_{1}}, t^{r_{2}}, \ldots, t^{r_{2}}, \ldots \ldots, t^{r_{s}}, \ldots, t^{r_{s}}\right),
$$

where the rational weight $r_{i}:=-\theta\left(d_{i}\right) / \alpha\left(d_{i}\right)$ appears $\left(d_{i}\right)_{v}$ times. 


\section{Hoskins}

Remark 3.7. For $\gamma \neq \gamma^{\prime}$, we note that the conjugacy classes of $\lambda_{\gamma}$ and $\lambda_{\gamma^{\prime}}$ are distinct.

Let $\|-\|_{\alpha}$ be the norm on 1-PSs of $G_{d}(Q)$ associated to $\alpha \in \mathbb{N}^{V}$ (cf. Example 2.4). For a quiver without relations, the following result is [Hos14, Theorem 5.5]. We can deduce the case with relations from this result using Theorem 2.8 and Remark 3.5.

TheOrem 3.8. Let $(Q, \mathcal{R})$ be a quiver with relations with dimension vector $d \in \mathbb{N}^{V}$, and let $\theta \in \mathbb{Z}^{V}$ and $\alpha \in \mathbb{N}^{V}$ be stability parameters such that $\sum_{v \in V} d_{v} \theta_{v}=0$. Then the HN stratification with respect to $(\theta, \alpha)$ and the Hesselink stratification with respect to $\rho_{\theta}$ and $\|-\|_{\alpha}$ coincide: if

$$
\operatorname{Rep}_{d}(Q, \mathcal{R})=\bigsqcup_{\gamma} R_{\gamma} \text { and } \operatorname{Rep}_{d}(Q, \mathcal{R})=\bigsqcup_{[\lambda]} S_{[\lambda]}
$$

denote these stratifications respectively, then $R_{\gamma}=S_{\left[\lambda_{\gamma}\right]}$.

In fact, in [Hos14], we show that this Hesselink stratification also coincides with a Morse-type stratification associated to the norm square of a moment map.

\subsection{Stratifications on the stack of quiver representations}

The affine scheme $\operatorname{Rep}_{d}(Q, \mathcal{R})$ parametrises a tautological family $\mathcal{W}$ of representations of $(Q, \mathcal{R})$ of dimension vector $d$ with the local universal property; thus, the collection of quiver representations of fixed dimension $d$ is bounded. This is in sharp contrast with the situation for coherent sheaves on a fixed projective scheme with fixed numerical invariants: this collection of sheaves is not bounded and so cannot be parametrised by a scheme (cf. Section 4). Moreover, the moduli stack $\mathcal{R e p}_{d}(Q, \mathcal{R})$ of representations of $(Q, \mathcal{R})$ of dimension $d$ is isomorphic to the global quotient stack

$$
\operatorname{Rep}_{d}(Q, \mathcal{R}) \cong\left[\operatorname{Rep}_{d}(Q, \mathcal{R}) / G_{d}(Q)\right] .
$$

As the Hesselink strata are $G_{d}(Q)$-invariant, we can take their stack quotients by $G_{d}(Q)$ to obtain a 'stacky Hesselink' stratification of $\mathcal{R e p}_{d}(Q, \mathcal{R})$ by locally closed substacks. Following Theorem 3.8, this stratification coincides with the stratification of the stack by HN types.

Corollary 3.9. On $\mathcal{R e p}_{d}(Q, \mathcal{R})$, the stacky Hesselink strata and the HN strata agree as stacks.

There are only finitely many HN types for representations of $Q$ of fixed dimension vector, and both stratifications are finite. In Section 4, we prove an analogous statement for coherent sheaves over a projective scheme; however, in this case, the stratifications are infinite and the stacky Hesselink stratification is obtained as a limit of (finite) Hesselink stratifications.

\section{Stratifications for moduli of sheaves}

Let $\left(X, \mathcal{O}_{X}(1)\right)$ be a projective scheme of finite type over $k$ with a very ample line bundle.

\subsection{Preliminaries}

For a sheaf $\mathcal{E}$ over $X$, we let $\mathcal{E}(n):=\mathcal{E} \otimes \mathcal{O}_{X}(n)$, and we let $P(\mathcal{E})$ denote the Hilbert polynomial of $\mathcal{E}$ with respect to $\mathcal{O}_{X}(1)$, whose value at $n \in \mathbb{N}$ is given by

$$
P(\mathcal{E}, n)=\chi(\mathcal{E}(n))=\sum_{i=0}^{n}(-1)^{i} \operatorname{dim} H^{i}(\mathcal{E}(n)) .
$$




\section{STRATIFICATIONS IN MODULI PROBLEMS}

The degree of $P(\mathcal{E})$ is equal to the dimension of $\mathcal{E}$ (that is, the dimension of the support of $\mathcal{E}$ ), and $\mathcal{E}$ is pure if all its non-zero subsheaves have the same dimension as $\mathcal{E}$. For a non-zero sheaf $\mathcal{E}$, the leading coefficient $r(\mathcal{E})$ of $P(\mathcal{E})$ is positive and we define the reduced Hilbert polynomial of $\mathcal{E}$ to be $P^{\text {red }}(\mathcal{E}):=P(\mathcal{E}) / r(\mathcal{E})$.

Definition 4.1 (Castelnuovo-Mumford [Mum66]). A sheaf $\mathcal{E}$ over $X$ is $n$-regular if

$$
H^{i}(\mathcal{E}(n-i))=0 \text { for all } i>0 \text {. }
$$

This is an open condition and, by Serre's vanishing theorem, any sheaf is $n$-regular for $n \gg 0$. Furthermore, any bounded family of sheaves is $n$-regular for $n \gg 0$.

Lemma 4.2 (cf. [Mum66]). For an $n$-regular sheaf $\mathcal{E}$ over $X$, we have the following results:

(i) The sheaf $\mathcal{E}$ is $m$-regular for all $m \geqslant n$.

(ii) The sheaf $\mathcal{E}(n)$ is globally generated with vanishing higher cohomology; that is, the evaluation map $H^{0}(\mathcal{E}(n)) \otimes \mathcal{O}_{X}(-n) \rightarrow \mathcal{E}$ is surjective and $H^{i}(\mathcal{E}(n))=0$ for $i>0$.

(iii) The natural multiplication maps $H^{0}(\mathcal{E}(n)) \otimes H^{0}\left(\mathcal{O}_{X}(m-n)\right) \rightarrow H^{0}(\mathcal{E}(m))$ are surjective for all $m \geqslant n$.

(iv) If $0 \rightarrow \mathcal{F}^{\prime} \rightarrow \mathcal{F} \rightarrow \mathcal{F}^{\prime \prime} \rightarrow 0$ is an exact sequence of sheaves over $X$ such that $\mathcal{F}^{\prime}$ and $\mathcal{F}^{\prime \prime}$ are both $n$-regular, then $\mathcal{F}$ is also $n$-regular.

\subsection{Construction of the moduli space of semistable sheaves}

In this section, we outline Simpson's construction [Sim94] of the moduli space of semistable sheaves on $\left(X, \mathcal{O}_{X}(1)\right)$ with Hilbert polynomial $P$. We define an ordering $\leqslant$ on rational polynomials in one variable by $P \leqslant Q$ if $P(x) \leqslant Q(x)$ for all sufficiently large $x$. For polynomials of a fixed degree with positive leading coefficient, this is equivalent to the lexicographic ordering on the coefficients.

Definition 4.3. A pure sheaf $\mathcal{F}$ over $X$ is semistable (in the sense of Gieseker) if for all non-zero subsheaves $\mathcal{E} \subset \mathcal{F}$, we have $P^{\operatorname{red}}(\mathcal{E}) \leqslant P^{\text {red }}(\mathcal{F})$.

By the Simpson-Le Potier bounds (cf. [Sim94, Theorem 1.1]), the set of semistable sheaves over $X$ with Hilbert polynomial $P$ is bounded; therefore, for $n \gg 0$, all semistable sheaves with Hilbert polynomial $P$ are $n$-regular.

Let $V_{n}:=k^{P(n)}$ be the trivial $P(n)$-dimensional vector space, and let $\operatorname{Quot}\left(V_{n} \otimes \mathcal{O}_{X}(-n), P\right)$ denote the Quot scheme parametrising quotients sheaves of $V_{n} \otimes \mathcal{O}_{X}(-n)$ with Hilbert polynomial $P$. Let $Q_{n}$ denote the open subscheme of this Quot scheme consisting of quotients $q: V_{n} \otimes \mathcal{O}_{X}(-n) \rightarrow \mathcal{E}$ such that $H^{0}(q(n))$ is an isomorphism. Every $n$-regular sheaf on $X$ with Hilbert polynomial $P$ can be represented as a quotient sheaf in $Q_{n}$ by choosing an isomorphism $H^{0}(\mathcal{E}(n)) \cong V_{n}$ (this is possible, as $\left.\operatorname{dim} H^{0}(\mathcal{E}(n))=P(n)\right)$ and using the surjective evaluation map $H^{0}(\mathcal{E}(n)) \otimes \mathcal{O}_{X}(-n) \rightarrow \mathcal{E}$. The group $G_{n}:=\operatorname{GL}\left(V_{n}\right)$ acts on $V_{n}$ and $Q_{n}$ in such a way that the $G_{n}$-orbits in $Q_{n}$ are in bijection with isomorphism classes of sheaves in $Q_{n}$. As the diagonal $\mathbb{G}_{m}$ acts trivially, we can consider the action of $\mathrm{SL}\left(V_{n}\right)$.

Let $Q_{n}^{\text {pure }} \subset Q_{n}$ denote the open subscheme consisting of quotient sheaves that are pure, and let $R_{n}$ denote the closure of $Q_{n}^{\text {pure }}$ in the Quot scheme. The $\operatorname{SL}\left(V_{n}\right)$-action on $R_{n}$ is linearised using Grothendieck's embedding of the Quot scheme into a Grassmannian by choosing $m \gg n$ : the corresponding line bundle is given by

$$
L_{n, m}:=\operatorname{det}\left(\pi_{*}\left(\mathcal{U}_{n} \otimes \pi_{X}^{*} \mathcal{O}_{X}(m)\right)\right),
$$




\section{Hoskins}

where $\mathcal{U}_{n}$ denotes the universal quotient sheaf on the Quot scheme and $\pi_{X}$ and $\pi$ denote the projection maps from $R_{n} \times X$ to $X$ and $R_{n}$, respectively.

Theorem 4.4 (Simpson [Sim94, Theorem 1.21]). Fix $\left(X, \mathcal{O}_{X}(1)\right)$ and a Hilbert polynomial $P$. Then, for $m \gg n \gg 0$, the moduli space of semistable sheaves on $X$ with Hilbert polynomial $P$ is a GIT quotient:

$$
M^{\mathrm{sS}}(X, P)=R_{n} / / L_{n, m} \mathrm{SL}\left(V_{n}\right) .
$$

In his proof, Simpson shows, for $m \gg n \gg 0$ (depending on $X$ and $P$ ), that an element $q: V_{n} \otimes \mathcal{O}_{X}(-n) \rightarrow \mathcal{E}$ in $R_{n}$ is GIT semistable if and only if $q$ belongs to the open subscheme $Q_{n}^{\text {ss }}$ of $Q_{n}$ consisting of quotient sheaves $q: V_{n} \otimes \mathcal{O}_{X}(-n) \rightarrow \mathcal{E}$ such that $\mathcal{E}$ is a semistable sheaf. In particular, the GIT semistable locus and the GIT quotient do not depend on $m$ and $n$ provided that these are taken sufficiently large.

\subsection{The Hesselink stratification of the Quot scheme}

For the action of $\operatorname{SL}\left(V_{n}\right)$ on $\operatorname{Quot}\left(V_{n} \otimes \mathcal{O}_{X}(-n), P\right)$ with respect to $L_{n, m}$ and the norm $\|-\|$ coming from the dot product for the diagonal torus $T \subset \operatorname{SL}\left(V_{n}\right)$ (cf. Example 2.4), there is a Hesselink stratification

$$
\operatorname{Quot}_{n}:=\operatorname{Quot}\left(V_{n} \otimes \mathcal{O}_{X}(-n), P\right)=\bigsqcup_{\beta \in \mathcal{B}\left(L_{n, m}\right)} S_{\beta} .
$$

In this section, for fixed $n$, we describe this stratification of Quot $_{n}$, we extend the results of [HK12] to non-pure sheaves and enhance the proof to a scheme-theoretic statement.

By Remark 2.10, the unstable indices $\beta$ can equivalently be viewed as conjugacy classes of rational 1-PSs $\left[\lambda_{\beta}\right]$ of $\operatorname{SL}\left(V_{n}\right)$. We choose a representative $\lambda_{\beta} \in X_{*}(T)$ and, by using the Weyl group action, we may assume that the weights are decreasing; that is,

$$
\lambda_{\beta}(t)=\operatorname{diag}\left(t^{r_{1}}, \ldots, t^{r_{1}}, t^{r_{2}}, \ldots, t^{r_{2}}, \ldots, t^{r_{s}}, \ldots, t^{r_{s}}\right),
$$

where $r_{1}, \ldots, r_{s}$ are strictly decreasing rational numbers such that $r_{i}$ occurs with multiplicity $l_{i}$. Thus, $\beta$ is equivalent to the decreasing sequence of rational weights $r(\beta):=\left(r_{1}, \ldots, r_{s}\right)$ with multiplicities $l(\beta):=\left(l_{1}, \ldots, l_{s}\right)$. Since $\lambda_{\beta}$ is a rational 1 -PS of $\operatorname{SL}\left(V_{n}\right)$, we note that

$$
\sum_{i=1}^{s} l_{i}=\operatorname{dim} V_{n}=P(n) \text { and } \sum_{i=1}^{s} r_{i} l_{i}=0 .
$$

We can describe the $\lambda_{\beta}$-fixed points as follows. The 1-PS $\lambda_{\beta}$ determines a filtration of $V:=V_{n}$

$$
0=V^{(0)} \subset V^{(1)} \subset \cdots \subset V^{(s)}=V, \text { where } V^{(i)}:=k^{l_{1}+\cdots+l_{i}}
$$

with $V^{i}:=V^{(i)} / V^{(i-1)}$ of dimension $l_{i}$. For a quotient $q: V \otimes \mathcal{O}_{X}(-n) \rightarrow \mathcal{E}$ in Quot, we let $\mathcal{E}^{(i)}:=q\left(V^{(i)} \otimes \mathcal{O}_{X}(-n)\right)$; then we have exact sequences of quotient sheaves



We fix an isomorphism $V \cong \oplus_{i=1}^{s} V^{i}$; then via this isomorphism, we have $\lim _{t \rightarrow 0} \lambda_{\beta}(t) \cdot q=\oplus_{i=1}^{s} q_{i}$ by [HL97, Lemma 4.4.3]. 
Lemma 4.5. For $\beta=([\lambda], d)$ with $r(\beta)=\left(r_{1}, \ldots, r_{s}\right)$ and $l(\beta)=\left(l_{1}, \ldots, l_{s}\right)$ defined following (4.1), we have

$$
\operatorname{Quot}^{\lambda}=\underset{\left(P_{1}, \ldots, P_{s}\right): \sum_{i=1}^{s} P_{i}=P}{ } \prod_{i=1}^{s} \operatorname{Quot}\left(V^{i} \otimes \mathcal{O}_{X}(-n), P_{i}\right) .
$$

Furthermore, let Quot $_{d}^{\lambda}$ be the union of connected components of the fixed point locus on which $\mu^{L_{n, m}}\left(-, \lambda_{\beta}\right)=-\left\|\lambda_{\beta}\right\|^{2}$; then

$$
\operatorname{Quot}_{d}^{\lambda} \cong \bigsqcup_{\substack{\left(P_{1}, \ldots, P_{s}\right): \sum_{i=1}^{s} P_{i}=P, \sum_{i=1}^{s} r_{i} P_{i}(m)+r_{i}^{2} l_{i}=0}} \prod_{i=1}^{s} \operatorname{Quot}\left(V^{i} \otimes \mathcal{O}_{X}(-n), P_{i}\right)
$$

Proof. The description of the $\lambda$-fixed locus follows from the discussion above. For a $\lambda$-fixed quotient sheaf $q: V \otimes \mathcal{O}_{X}(-n) \rightarrow \oplus_{i=1}^{s} \mathcal{E}^{i}$, we have that $\mu\left(q, \lambda_{\beta}\right)=\sum_{i=1}^{s} r_{i} P\left(\mathcal{E}^{i}, m\right)$ by [HL97, Lemma 4.4.4]. The second statement then follows as $\left\|\lambda_{\beta}\right\|^{2}=\sum_{i=1}^{s} r_{i}^{2} l_{i}$.

By Theorem 2.8, for $\beta=([\lambda], d)$ as above, the associated unstable stratum $S_{\beta}$ can be constructed from the limit set $Z_{d}^{\lambda}$ which is the GIT semistable set for the subgroup

$$
G_{\lambda} \cong\left\{\left(g_{1}, \ldots, g_{s}\right) \in \prod_{i=1}^{s} \mathrm{GL}\left(V^{i}\right): \prod_{i=1}^{s} \operatorname{det} g_{i}=1\right\}
$$

acting on Quot $_{d}^{\lambda}$ with respect to the linearisation $L_{\beta}$ obtained by twisting $L_{n, m}$ by the (rational) character $\chi_{\beta}: G_{\lambda} \rightarrow \mathbb{G}_{m}$, where

$$
\chi_{\beta}\left(g_{1}, \ldots, g_{s}\right)=\prod_{i=1}^{s}\left(\operatorname{det} g_{i}\right)^{r_{i}} .
$$

Proposition 4.6. Let $\beta=([\lambda], d)$ with $r(\beta)=\left(r_{1}, \ldots, r_{s}\right)$ and $l(\beta)=\left(l_{1}, \ldots, l_{s}\right)$ as above; then

$$
Z_{d}^{\lambda} \cong \bigsqcup_{\left(P_{1}, \ldots, P_{s}\right)} \prod_{i=1}^{s} \operatorname{Quot}\left(V^{i} \otimes \mathcal{O}_{X}(-n), P_{i}\right)^{\mathrm{SL}\left(V^{i}\right) \text {-ss }}\left(L_{n, m}\right)
$$

where the union is taken over tuples $\left(P_{1}, \ldots, P_{s}\right)$ such that $\sum_{i=1}^{s} P_{i}=P$ and

$$
r_{i}=\frac{P(m)}{P(n)}-\frac{P_{i}(m)}{l_{i}} \quad \text { for } i=1, \ldots, s .
$$

Proof. The centre $Z\left(G_{\lambda}\right) \cong\left\{\left(t_{1}, \ldots, t_{s}\right) \in\left(\mathbb{G}_{m}\right)^{s}: \prod_{i=1}^{s} t_{i}^{l_{i}}=1\right\}$ acts trivially on Quot ${ }^{\lambda}$ and acts on the fibre of $L$ over $q \in$ Quot $^{\lambda}$, lying in a component indexed by $\left(P_{1}, \ldots, P_{s}\right)$, by a character

$$
\chi_{q}: Z\left(G_{\lambda}\right) \rightarrow \mathbb{G}_{m} \quad \text { given by } \chi_{q}\left(t_{1}, \ldots, t_{s}\right)=\prod_{i=1}^{s} t_{i}^{P_{i}(m)} .
$$

Therefore, $Z\left(G_{\lambda}\right)$ acts on the fibre of $L_{\beta}$ over $q$ by $\chi_{q} \chi_{\beta}\left(t_{1}, \ldots, t_{s}\right)=\prod_{i=1}^{s} t_{i}^{P_{i}(m)+r_{i} l_{i}}$. By the Hilbert-Mumford criterion, $q$ is unstable for the action of $G_{\lambda}$ with respect to $L_{\beta}$ unless $\chi_{q} \chi_{\beta}$ is trivial; that is, there is a constant $C$ such that $C l_{i}=P_{i}(m)+r_{i} l_{i}$ for $i=1, \ldots, s$. In this case,

$$
C P(n)=\sum_{i=1}^{s} C l_{i}=\sum_{i=1}^{s} P_{i}(m)+r_{i} l_{i}=P(m),
$$




\section{Hoskins}

and we see that the conditions given at (4.2) are necessary for $q$ to belong to $Z_{d}^{\lambda}$.

We suppose that $\left(P_{1}, \ldots, P_{s}\right)$ is a tuple satisfying $\sum_{i=1}^{s} P_{i}=P$ and the conditions given at (4.2). Since $G_{\lambda}$ is, modulo a finite group, $\prod_{i=1}^{s} \mathrm{SL}\left(V^{i}\right) \times Z\left(G_{\lambda}\right)$ and $Z\left(G_{\lambda}\right)$ acts on both

$$
\prod_{i=1}^{s} \operatorname{Quot}\left(V^{i} \otimes \mathcal{O}_{X}(-n), P_{i}\right)
$$

and $L_{\beta}$ trivially, the semistable locus for $G_{\lambda}$ is equal to the semistable locus for $\prod_{i=1}^{s} \operatorname{SL}\left(V^{i}\right)$. As $\prod_{i=1}^{s} \mathrm{SL}\left(V^{i}\right)$-linearisations, we have that $L_{\beta}=L$. It then follows that

$$
\left(\prod_{i=1}^{s} \operatorname{Quot}\left(V^{i} \otimes \mathcal{O}_{X}(-n), P_{i}\right)\right)^{\prod_{i=1}^{s} \mathrm{SL}\left(V^{i}\right) \text {-ss }}(L)=\prod_{i=1}^{s} \operatorname{Quot}\left(V^{i} \otimes \mathcal{O}_{X}(-n), P_{i}\right)^{\mathrm{SL}\left(V^{i}\right) \text {-ss }}(L)
$$

(see, for example, [HK12, Lemma 6.6]), which completes the proof of the proposition.

The following corollary is an immediate consequence of Proposition 4.6 and Theorem 2.8.

Corollary 4.7. For $\beta$ as at (4.1), the scheme $S_{\beta}$ parametrises quotients $q: V \otimes \mathcal{O}_{X}(-n) \rightarrow \mathcal{E}$ with a filtration $0=W^{(0)} \subset W^{(1)} \subset \cdots \subset W^{(s)}=V$ such that, for $i=1, \ldots, s$, we have

(i) $\operatorname{dim} W^{i}=l_{i}$, where $W^{i}:=W^{(i)} / W^{(i-1)}$;

(ii) $P(n) P\left(\mathcal{E}^{i}, m\right)=l_{i}\left(P(m)-r_{i} P(n)\right)$; and

(iii) the quotient sheaf $q^{i}: W^{i} \otimes \mathcal{O}_{X}(-n) \rightarrow \mathcal{E}^{i}$ is $\mathrm{SL}\left(W^{i}\right)$-semistable with respect to $L_{n, m}$,

where $\mathcal{E}^{(i)}:=q\left(W^{(i)} \otimes \mathcal{O}_{X}(-n)\right)$ and $\mathcal{E}^{i}:=\mathcal{E}^{(i)} / \mathcal{E}^{(i-1)}$. Furthermore, $S_{\beta}=\mathrm{SL}(V) \cdot Y_{d}^{\lambda}$, where $Y_{\lambda}^{d}$ parametrises quotients $q$ with a filtration $W^{\bullet}$ as above such that $W^{\bullet}=V^{\bullet}$.

Remark 4.8. As already noted, the Hesselink strata may not be connected, and so it is natural to further decompose the limit sets $Z_{d}^{\lambda}$ using tuples of Hilbert polynomials $\nu=\left(P_{1}, \ldots, P_{s}\right)$; that is, we can write

$$
Z_{d}^{\lambda}=\bigsqcup_{\nu \in \mathcal{C}_{\beta}} Z_{d, \nu}^{\lambda}, \quad \text { where } Z_{d, \nu}^{\lambda} \cong \prod_{i=1}^{s} \operatorname{Quot}\left(V^{i} \otimes \mathcal{O}_{X}(-n), P_{i}\right)^{\operatorname{SL}\left(V^{i}\right)-\mathrm{ss}}\left(L_{n, m}\right)
$$

for $\nu=\left(P_{1}, \ldots, P_{s}\right)$, as a union of connected components of $Z_{d}^{\lambda}$. Here the index set $\mathcal{C}_{\beta}$ is the set of tuples $\nu=\left(P_{1}, \ldots, P_{s}\right)$ such that $\sum_{i=1}^{s} P_{i}=P$, the conditions (4.2) hold, and $Z_{d, \nu}^{\lambda}$ is non-empty. Then $S_{\beta}$ is a disjoint union of the refined strata $S_{\beta, \nu}:=\mathrm{SL}(V) \cdot p_{\lambda}^{-1}\left(Z_{d, \nu}^{\lambda}\right)$ indexed by $\beta \in \mathcal{C}_{\beta}$. This gives a refinement of the Hesselink stratification

$$
\operatorname{Quot}\left(V \otimes \mathcal{O}_{X}(-n), P\right)=\bigsqcup_{\substack{\beta \in \mathcal{B} \\ \nu \in \mathcal{C}_{\beta}}} S_{\beta, \nu}
$$

We note that we may have strata $S_{\beta, \nu}$ and $S_{\beta^{\prime}, \nu}$ with different Hesselink indices $\beta$ and $\beta^{\prime}$ but the same tuple of Hilbert polynomials $\nu$. However, we will later see in Definition 4.18 that for a tuple $\nu$, there is a natural associated Hesselink index $\beta_{n, m}(\nu)$ depending on $m \gg n \gg 0$.

\subsection{HN filtrations for coherent sheaves}

In this section, we describe a canonical destabilising filtration for each coherent sheaf, known as its Harder-Narasimhan (HN) filtration [HN75]. 


\section{STRATIFICATIONS IN MODULI PROBLEMS}

Definition 4.9. A pure HN filtration of a sheaf $\mathcal{F}$ is a filtration by subsheaves

$$
0=\mathcal{F}^{(0)} \subset \mathcal{F}^{(1)} \subset \cdots \subset \mathcal{F}^{(s)}=\mathcal{F}
$$

such that the $\mathcal{F}_{i}:=\mathcal{F}^{(i)} / \mathcal{F}^{(i-1)}$ are semistable and $P^{\text {red }}\left(\mathcal{F}_{1}\right)>P^{\text {red }}\left(\mathcal{F}_{2}\right)>\cdots>P^{\text {red }}\left(\mathcal{F}_{s}\right)$.

By [HL97, Theorem 1.3.4], every pure sheaf has a unique pure HN filtration. To define HN filtrations for non-pure sheaves, we need an alternative definition of semistability that does not involve reduced Hilbert polynomials, as every non-pure sheaf $\mathcal{E}$ has a non-zero subsheaf whose Hilbert polynomial has degree strictly less than that of $\mathcal{E}$. For this, we use an extended notion of semistability due to Rudakov [Rud97]. We define a partial ordering $\preccurlyeq$ on $\mathbb{Q}[x]$ by

$$
P \preccurlyeq Q \Longleftrightarrow \frac{P(n)}{P(m)} \leqslant \frac{Q(n)}{Q(m)} \text { for } m \gg n \gg 0
$$

and similarly define a strict partial order $\prec$ by replacing $\leqslant$ with $<$. This ordering allows us to compare polynomials with positive leading coefficient of different degrees in a way that polynomials of lower degree are larger with respect to this ordering.

Remark 4.10. Rudakov formulated this preordering using the coefficients of the polynomials: for rational polynomials $P(x)=p_{d} x^{d}+\cdots+p_{0}$ and $Q(x)=q_{e} x^{e}+\cdots+q_{0}$, let

$$
\Lambda(P, Q):=\left(\lambda_{f, f-1}, \ldots, \lambda_{f, 0}, \lambda_{f-1, f-2}, \ldots, \lambda_{f-1,0}, \ldots, \lambda_{1,0}\right), \quad \text { where } \lambda_{i, j}:=p_{i} q_{j}-q_{i} p_{j}
$$

and $f:=\max (d, e)$. We say $\Lambda(P, Q)>0$ if the first non-zero $\lambda_{i, j}$ appearing in $\Lambda(P, Q)$ is positive. Then $P \preccurlyeq Q$ is equivalent to $\Lambda(P, Q) \geqslant 0$.

Definition 4.11. A sheaf $\mathcal{F}$ is semistable if $P(\mathcal{E}) \preccurlyeq P(\mathcal{F})$ for all non-zero subsheaves $\mathcal{E} \subset \mathcal{F}$.

This definition of semistability implies purity, as polynomials of smaller degree are larger with respect to $\preccurlyeq$. Moreover, for Hilbert polynomials $P(\mathcal{E})$ and $P(\mathcal{F})$ of the same degree, we have $P(\mathcal{E}) \preccurlyeq P(\mathcal{F})$ if and only if $P^{\text {red }}(\mathcal{E}) \leqslant P^{\text {red }}(\mathcal{F})$. Thus, a sheaf is semistable in the sense of Definition 4.11 if and only if it is semistable in the sense of Definition 4.3.

Definition 4.12. A HN filtration of a sheaf $\mathcal{E}$ is a filtration by subsheaves

$$
0=\mathcal{E}^{(0)} \subset \mathcal{E}^{(1)} \subset \cdots \subset \mathcal{E}^{(s)}=\mathcal{E}
$$

such that the $\mathcal{E}_{i}:=\mathcal{E}^{(i)} / \mathcal{E}^{(i-1)}$ are semistable and $P\left(\mathcal{E}_{1}\right) \succ P\left(\mathcal{E}_{2}\right) \succ \cdots \succ P\left(\mathcal{E}_{s}\right)$.

Using this definition of HN filtration, every coherent sheaf has a unique HN filtration (cf. [Rud97, Corollary 28]). We can construct the HN filtration of a sheaf from its torsion filtration and the pure HN filtrations of the subquotients in the torsion filtration as follows.

Proposition 4.13. Let $0 \subset T^{(0)}(\mathcal{E}) \subset \cdots \subset T^{(d)}(\mathcal{E})=\mathcal{E}$ be the torsion filtration of $\mathcal{E}$, and let

$$
0=\mathcal{F}_{i}^{(0)} \subset \mathcal{F}_{i}^{(1)} \subset \cdots \subset \mathcal{F}_{i}^{\left(s_{i}\right)}=T_{i}(\mathcal{E}):=T^{(i)}(\mathcal{E}) / T^{(i-1)}(\mathcal{E})
$$

be the pure $H N$ filtrations of the subquotients in the torsion filtration. Then $\mathcal{E}$ has HN filtration

$$
0=\mathcal{E}_{0}^{(0)} \subset \mathcal{E}_{0}^{(1)} \subset \mathcal{E}_{1}^{(1)} \subset \cdots \subset \mathcal{E}_{1}^{\left(s_{1}\right)} \subset \cdots \subset \mathcal{E}_{d}^{(1)} \subset \cdots \subset \mathcal{E}_{d}^{\left(s_{d}\right)}=\mathcal{E},
$$

where $\mathcal{E}_{i}^{(j)}$ is the preimage of $\mathcal{F}_{i}^{(j)}$ under the quotient map $T^{(i)}(\mathcal{E}) \rightarrow T_{i}(\mathcal{E})$.

Proof. As the HN filtration is unique, it suffices to check that the subquotients are semistable with decreasing Hilbert polynomials for $\prec$. First, we note that $\mathcal{E}_{i}^{(0)}=T^{(i-1)}(\mathcal{E})=\mathcal{E}_{i-1}^{\left(s_{i-1}\right)}$ and

$$
\mathcal{E}_{i}^{j}:=\mathcal{E}_{i}^{(j)} / \mathcal{E}_{i}^{(j-1)} \cong \mathcal{F}_{i}^{(j)} / \mathcal{F}_{i}^{(j-1)}=: \mathcal{F}_{i}^{j}
$$




\section{Hoskins}

Since (4.3) is the pure HN filtration of $T_{i}(\mathcal{E})$, we have inequalities $P^{\text {red }}\left(\mathcal{F}_{i}^{1}\right)>\cdots>P^{\text {red }}\left(\mathcal{F}_{i}^{s_{i}}\right)$ and the subquotients $\mathcal{F}_{i}^{j}$ are semistable. It follows that

$$
P\left(\mathcal{E}_{0}^{1}\right) \succ P\left(\mathcal{E}_{1}^{1}\right) \succ \cdots \succ P\left(\mathcal{E}_{1}^{s_{1}}\right) \succ \cdots \succ P\left(\mathcal{E}_{d}^{1}\right) \succ \cdots \succ P\left(\mathcal{E}_{d}^{s_{d}}\right),
$$

as $\operatorname{deg} P\left(\mathcal{F}_{i}^{j}\right)=i$ and polynomials of lower degree are larger with respect to this ordering.

DEFINITION 4.14. Let $\mathcal{E}$ be a sheaf with HN filtration $0=\mathcal{E}^{(0)} \subset \mathcal{E}^{(1)} \subset \cdots \subset \mathcal{E}^{(s)}=\mathcal{E}$; then the HN type of $\mathcal{E}$ is the tuple $\tau(\mathcal{E}):=\left(P\left(\mathcal{E}_{1}\right), \ldots, P\left(\mathcal{E}_{s}\right)\right)$, where $\mathcal{E}_{i}:=\mathcal{E}^{(i)} / \mathcal{E}^{(i-1)}$. We say that $\tau=\left(P_{1}, \ldots, P_{s}\right)$ is a pure HN type if all polynomials $P_{i}$ have the same degree.

\subsection{The HN stratification of the stack of coherent sheaves}

Let $\mathcal{C o h}_{P}(X)$ denote the stack of sheaves on $X$ with Hilbert polynomial $P$; this is an Artin stack such that

$$
\operatorname{Coh}_{P}(X) \cong \bigcup_{n}\left[Q_{n}^{o} / G_{n}\right]
$$

where $G_{n}=\mathrm{GL}\left(V_{n}\right)$ and $Q_{n}^{o}$ is the open subscheme of $\operatorname{Quot}\left(V_{n} \otimes \mathcal{O}_{X}(-n), P\right)$ consisting of quotient sheaves $q: V_{n} \otimes \mathcal{O}_{X}(-n) \rightarrow \mathcal{E}$ such that $H^{0}(q(n))$ is an isomorphism and $H^{i}(\mathcal{E}(n))=0$ for $i>0$ (cf. [LM00, Theorem 4.6.2.1]).

Let $\operatorname{HNT}(X, P)$ denote the set of HN types of sheaves over $X$ with Hilbert polynomial $P$. Thus, if $\tau=\left(P_{1}, \ldots, P_{s}\right) \in \operatorname{HNT}(X, P)$, then $\sum_{i=1}^{s} P_{i}=P$ and $P_{1} \succ P_{2} \succ \cdots \succ P_{s}$ and, moreover, there exists a sheaf with this HN type. For such a HN type $\tau$, we define the $(n, m)$ th Shatz polygon $\Gamma(\tau, n, m)$ to be the union of the line segments joining $x_{k}:=\left(\sum_{j=1}^{k} P_{j}(m), \sum_{j=1}^{k} P_{j}(n)\right)$ to $x_{k+1}$ for $k=0, \ldots, s-1$. We define a partial order $\leqslant$ on $\operatorname{HNT}(X, P)$ by $\tau \leqslant \tau^{\prime}$ if $\Gamma(\tau, n, m)$ lies above $\Gamma\left(\tau^{\prime}, n, m\right)$ for $m \gg n \gg 0$.

Theorem 4.15 (Shatz [Sha77] and Nitsure [Nit11]). Let $\mathcal{F}$ be a family of sheaves on $X$ with Hilbert polynomial $P$ parametrised by a scheme $S$; then the $H N$ type function $S \rightarrow \operatorname{HNT}(X, P)$ given by $s \mapsto \tau\left(\mathcal{F}_{s}\right)$ is upper semicontinuous. Furthermore,

(i) the set $S_{>\tau}=\left\{s \in S: \tau\left(\mathcal{F}_{s}\right)>\tau\right\}$ is closed in $S$;

(ii) the set $S_{\tau}=\left\{s \in S: \tau\left(\mathcal{F}_{s}\right)=\tau\right\}$ is locally closed in $S$;

(iii) the set $S_{\tau}$ has a unique scheme structure with the following property: a morphism $T \rightarrow S$ factors via $S_{\tau}$ if and only if the pullback $\mathcal{F}_{T}$ of $\mathcal{F}$ to $X \times T$ has a filtration by coherent sheaves whose successive quotients are flat over $T$ and for each $t \in T$, this filtration gives the HN filtration of $\left(\mathcal{F}_{T}\right)_{t}$, which is of type $\tau$;

(iv) there is a finite $H N$ (or Shatz) stratification of $S$ into disjoint subschemes $S_{\tau}$ such that

$$
\overline{S_{\tau}} \subset \bigsqcup_{\tau^{\prime} \geqslant \tau} S_{\tau^{\prime}}
$$

The universal quotient sheaf $\mathcal{U}_{n}$ over $X \times Q_{n}^{o}$ is a family of sheaves on $X$ with Hilbert polynomial $P$ parametrised by $Q_{n}^{o}$; therefore, we have an associated HN stratification

$$
Q_{n}^{o}=\bigsqcup_{\tau} Q_{n, \tau}
$$

As the HN strata $Q_{n, \tau}$ are $G_{n}$-invariant, this stratification descends to the stack quotient

$$
\left[Q_{n}^{o} / G_{n}\right]=\bigsqcup_{\tau}\left[Q_{n, \tau} / G_{n}\right]
$$

From the description (4.4) of $\mathcal{C} \mathrm{oh}_{P}(X)$, we have the following corollary. 


\section{STRATIFICATIONS IN MODULI PROBLEMS}

COROLLARY 4.16. There is a HN stratification on the stack of coherent sheaves

$$
\operatorname{Coh}_{P}(X)=\bigsqcup_{\tau \in \operatorname{HNT}(X, P)} \mathcal{C o h}_{P}^{\tau}(X)
$$

into disjoint locally closed substacks $\mathcal{C o h}_{P}^{\tau}(X)$ such that $\overline{\mathcal{C o h}_{P}^{\tau}(X)} \subset \bigsqcup_{\tau^{\prime} \geqslant \tau} \mathcal{C o h}_{P}^{\tau^{\prime}}(X)$.

Remark 4.17. If $\tau=(P)$ is the trivial HN type, then $\mathcal{C o h}_{X, P}^{\tau}=\mathcal{C o h}_{X, P}^{\mathrm{ss}}$ and for $n \gg 0$,

$$
\mathcal{C o h}_{X, P}^{\mathrm{ss}} \cong\left[Q_{n}^{\mathrm{ss}} / G_{n}\right]
$$

where $Q_{n}^{\text {ss }}=Q_{n,(P)}$ is an open subscheme of $\operatorname{Quot}\left(V_{n} \otimes \mathcal{O}_{X}(-n), P\right)$. In fact, an analogous statement holds for all HN types (cf. Proposition 4.21).

\subsection{The Hesselink and HN stratifications}

In this section, we prove, for each HN type $\tau$, that the HN stratum $Q_{n, \tau}$ is contained in a Hesselink stratum of $\operatorname{Quot}\left(V_{n} \otimes \mathcal{O}_{X}(-n), P\right)$ for $m \gg n \gg 0$; this generalises an analogous result for pure $\mathrm{HN}$ types given in [HK12].

Definition 4.18. For a tuple of Hilbert polynomials $\nu=\left(P_{1}, \ldots, P_{s}\right)$ which sum to $P$ and natural numbers $(n, m)$, we let $\beta_{n, m}(\nu)$ denote the conjugacy class of the rational 1-PS

$$
\lambda_{\beta_{n, m}(\nu)}(t)=\operatorname{diag}(\underbrace{t^{r_{1}}, \ldots, t^{r_{1}}}_{P_{1}(n)}, \ldots, \underbrace{t^{r_{s}}, \ldots, t^{r_{s}}}_{P_{s}(n)}), \quad \text { where } r_{i}:=\frac{P(m)}{P(n)}-\frac{P_{i}(m)}{P_{i}(n)} .
$$

If $\nu=\tau$ is a HN type, we have $P_{1} \succ P_{2} \succ \cdots \succ P_{s}$ and thus for $m \gg n \gg 0$,

$$
\frac{P_{1}(n)}{P_{1}(m)}>\frac{P_{2}(n)}{P_{2}(m)}>\cdots>\frac{P_{s}(n)}{P_{s}(m)} ;
$$

that is, the weights $r_{i}$ in this rational 1-PS are decreasing.

The following result simultaneously enhances and generalises a joint result with Kirwan in [HK12]. More precisely, [HK12, Proposition 6.8] states that for a pure HN type $\tau$ (that is, a HN type of a pure sheaf) and for $m \gg n \gg 0$, the HN stratum of a closed subscheme $R_{n} \subset$ Quot $_{n}$ is contained in the Hesselink stratum in $R_{n}$ indexed by $\beta_{n, m}(\tau)$. The proof of loc. cit. was set-theoretic, whereas the proof below is scheme-theoretic and applies to all HN types.

Theorem 4.19. For $\tau=\left(P_{1}, \ldots, P_{s}\right) \in \operatorname{HNT}(X, P)$ and $m \gg n \gg 0$, the HN stratum $Q_{n, \tau}$ is a closed subscheme of the stratum $S_{\beta_{n, m}(\tau)}$ for the Hesselink stratification of Quot ${ }_{n}$ with respect to $L_{n, m}$. Moreover, $Q_{n, \tau}$ is a closed subscheme of the refined stratum $S_{\beta_{n, m}(\tau), \tau}$.

Proof. We take $n \gg 0$ such that all semistable sheaves with Hilbert polynomial $P_{i}$ are $n$-regular for $i=1, \ldots, s$. Then every sheaf with HN type $\tau$ is $n$-regular, as it admits a filtration whose successive quotients are $n$-regular, and so can be parametrised by the HN stratum $Q_{n, \tau}$.

Let $V_{n}^{i}:=k^{P_{i}(n)}$, and let $Q_{n}^{i} \subset \operatorname{Quot}\left(V_{n}^{i} \otimes \mathcal{O}_{X}(-n), P_{i}\right)$ be the open subscheme consisting of quotients $q$ : $V_{n}^{i} \otimes \mathcal{O}_{X}(-n) \rightarrow \mathcal{E}^{i}$ such that $H^{0}(q(n))$ is an isomorphism. Let $\left(Q_{n}^{i}\right)$ pure be the open subscheme of $Q_{n}^{i}$ parametrising pure sheaves, and let $R_{n}^{i}$ be the closure of this subscheme in the Quot scheme. By [Sim94, Theorem 1.19], we can take $m \gg n$ such that the GIT semistable set for $\operatorname{SL}\left(V_{n}^{i}\right)$ acting on $R_{n}^{i}$ with respect to the linearisation $L_{n, m}$ is the lowest HN stratum in $Q_{n}^{i}$ parametrising semistable sheaves; that is,

$$
\left(R_{n}^{i}\right)^{\mathrm{SL}\left(V_{n}^{i}\right)-\mathrm{ss}}\left(L_{n, m}\right)=\left(Q_{n}^{i}\right)^{\mathrm{ss}}:=\left(Q_{n}^{i}\right)_{\left(P_{i}\right)}
$$




\section{Hoskins}

for $i=1, \ldots, s$. Furthermore, we assume $m \gg n \gg 0$, so that the inequalities (4.6) hold.

As described above, the index $\beta=\beta_{n, m}(\tau)=([\lambda], d)$ determines a filtration $0=V_{n}^{(0)} \subset \cdots \subset$ $V_{n}^{(s)}=V_{n}$, where $V_{n}^{i}:=V_{n}^{(i)} / V_{n}^{(i-1)}=k^{P_{i}(n)}$. By construction, the conditions (4.2) hold for $r(\beta)$ and $l(\beta)$; hence

$$
Z_{n, m}(\tau):=\prod_{i=1}^{s}\left(Q_{n}^{i}\right)^{\mathrm{ss}} \subset \prod_{i=1}^{s} \operatorname{Quot}\left(V_{n}^{i} \otimes \mathcal{O}_{X}(-n), P_{i}\right)^{\mathrm{SL}\left(V_{n}^{i}\right) \text {-ss }}\left(L_{n, m}\right) \cong Z_{d, \tau}^{\lambda} \subset Z_{d}^{\lambda} .
$$

Both inclusions are closed inclusions: the first as $R_{n}^{i}$ is closed in $\operatorname{Quot}\left(V_{n}^{i} \otimes \mathcal{O}_{X}(-n), P_{i}\right)$ and the second as $Z_{d, \tau}^{\lambda}$ is closed in $Z_{d}^{\lambda}$. Therefore, associated to this closed subscheme $\left(Z_{d}^{\lambda}\right)^{\prime}:=Z_{n, m}(\tau)$ of the limit set $Z_{d}^{\lambda}$, there is a closed subscheme $S_{\beta}^{\prime}=\operatorname{SL}\left(V_{n}\right) \cdot p_{\lambda}^{-1}\left(\left(Z_{d}^{\lambda}\right)^{\prime}\right)$ of the Hesselink strata $S_{\beta}$. In fact, $S_{\beta}^{\prime}$ is a closed subscheme of the refined Hesselink stratum $S_{\beta, \tau}$ as $\left(Z_{d}^{\lambda}\right)^{\prime}$ is a closed subscheme of $Z_{d, \tau}^{\lambda}$.

To complete the proof, we need to show that the schemes $Q_{n, \tau}$ and $S_{\beta}^{\prime}$ coincide. We first show that these agree set-theoretically and then use the universal property of the scheme structure on $Q_{n, \tau}$ given by Theorem 4.15 (iii) to prove the scheme structures agree. By construction, a quotient sheaf $q: V_{n} \otimes \mathcal{O}_{X}(-n) \rightarrow \mathcal{F}$ belongs to $p_{\lambda}^{-1}\left(Z_{n, m}(\tau)\right)$ if for the filtration

$$
0 \subset \mathcal{F}^{(1)} \subset \cdots \subset \mathcal{F}^{(s)}=\mathcal{F}, \quad \text { where } \mathcal{F}^{(i)}:=q\left(V_{n}^{(i)} \otimes \mathcal{O}_{X}(-n)\right),
$$

the successive quotients $\mathcal{F}^{(i)} / \mathcal{F}^{(i-1)}$ are semistable with Hilbert polynomial $P_{i}$. Therefore, these quotient sheaves have HN type $\tau$. Since the $\mathrm{SL}\left(V_{n}\right)$-action does not change the HN type, we have a set-theoretic inclusion $S_{\beta}^{\prime}:=\mathrm{SL}\left(V_{n}\right) \cdot p_{\lambda}^{-1}\left(Z_{n, m}(\tau)\right) \subset Q_{n, \tau}$. Conversely, for a point $q: V_{n} \otimes$ $\mathcal{O}_{X}(-n) \rightarrow \mathcal{F}$ in $Q_{n, \tau}$ with HN filtration given by

$$
0=\mathcal{F}^{(0)} \subset \mathcal{F}^{(1)} \subset \cdots \subset \mathcal{F}^{(s)}=\mathcal{F}
$$

we can choose a $g \in \mathrm{SL}\left(V_{n}\right)$ that sends the filtration $V_{n}^{(i)}$ to $W_{n}^{(i)}:=H^{0}(q(n))^{-1}\left(H^{0}\left(\mathcal{F}^{(i)}(n)\right)\right)$; then $g \cdot q \in p_{\lambda}^{-1}\left(Z_{n, m}(\tau)\right)$. Hence, as sets, $Q_{n, \tau}$ and $S_{\beta}^{\prime}$ agree.

By Theorem 4.15(iii), to show that the scheme structures coincide, we need to show that a morphism $T \rightarrow Q_{n}^{o}$ factors through $S_{\beta}^{\prime}$ if and only if the pullback $\mathcal{U}_{T}$ of the universal quotient sheaf $\mathcal{U}:=\mathcal{U}_{n}$ over $Q_{n}^{o} \times X$ to $T \times X$ has a relative $\mathrm{HN}$ filtration of type $\tau$ (that is, a filtration whose successive quotients are flat families of sheaves over $T$ which are semistable and have Hilbert polynomials given by the $P_{i}$ ). First of all, we note that there is a filtration of the restriction of $\mathcal{U}_{n}$ to $X \times S_{\beta}^{\prime}$ induced by the filtration of $\mathcal{U}$ over $X \times p_{\lambda}^{-1}\left(Z_{n, m}(\tau)\right)$ given by

$$
\mathcal{U}^{(i)}:=q\left(V_{n}^{(i)} \otimes \pi_{X}^{*} \mathcal{O}_{X}(-n)\right),
$$

where $q$ denotes the restriction of the universal quotient homomorphism to $X \times p_{\lambda}^{-1}\left(Z_{n, m}(\tau)\right)$. Moreover, this is a filtration of $\mathrm{HN}$ type $\tau$. In particular, if a morphism $T \rightarrow Q_{n}^{o}$ factors through $S_{\beta}^{\prime}$, then the pullback $\mathcal{U}_{T}$ of the universal family to $X \times T$ admits a filtration $\mathcal{U}_{T}^{(i)}$ of $\mathrm{HN}$ type $\tau$.

Conversely, suppose that we have a morphism $f: T \rightarrow Q_{n}^{o}$ such that $\mathcal{U}_{T}$ has a relative HN filtration

$$
0=\mathcal{U}_{T}^{(0)} \subset \mathcal{U}_{T}^{(1)} \subset \cdots \subset \mathcal{U}_{T}^{(s)}=\mathcal{U}_{T}
$$

of type $\tau$. Then to complete the proof, we need to show that the morphism $f$ factors via the locally closed immersion $S_{\beta}^{\prime} \hookrightarrow Q_{n}^{o}$. In particular, we note that any such factorisation is unique. The morphism $f$ from $T$ to the Quot scheme is equivalent to a family of quotient sheaves parametrised 


\section{STRATIFICATIONS IN MODULI PROBLEMS}

by $T$, that is, a surjective homomorphism of sheaves over $X \times T$

$$
q_{T}: V_{n} \otimes \pi_{X}^{*} \mathcal{O}_{X}(-n) \rightarrow \mathcal{U}_{T},
$$

where $\pi_{X}: X \times T \rightarrow X$ denotes the projection. Since $f$ has image in $Q_{n}^{o}$, we have, for each $t \in T$,

(1) $H^{j}\left(X, \mathcal{U}_{T, t}(n)\right)=0$ for $j>0$;

(2) $H^{0}\left(q_{T, t}(n)\right): V_{n} \rightarrow H^{0}\left(\mathcal{U}_{T, t}(n)\right)$ is an isomorphism.

Let $\pi_{T}: X \times T \rightarrow T$ be the projection morphism. Then statement (1) implies that $\pi_{T *}\left(\mathcal{U}_{T}(n)\right)$ is a locally free sheaf of rank $P(n)$ by [GD63, Corollaire 7.9.9], and statement (2) implies that the homomorphism of locally free sheaves over $T$

$$
V_{n} \otimes \mathcal{O}_{T} \cong V_{n} \otimes \pi_{T *} \pi_{X}^{*} \mathcal{O}_{X} \rightarrow\left(\pi_{T}\right)_{*}\left(\mathcal{U}_{T}(n)\right)
$$

is an isomorphism. Our choice of $n$ implies that any semistable sheaf over $X$ with Hilbert polynomial $P_{i}$ is $n$-regular, and, as the extension of two $n$-regular sheaves is also $n$-regular (see Lemma 4.2), it follows that the successive subsheaves appearing in the HN filtration of a sheaf with HN type $\tau$ are $n$-regular. Consequently, for $1 \leqslant i \leqslant s$, we have $H^{j}\left(\mathcal{U}_{T, t}^{(i)}(n)\right)=0$ for $j>0$ and so $\pi_{T *}\left(\mathcal{U}_{T}^{(i)}(n)\right)$ is a locally free sheaf of $\operatorname{rank} P^{(i)}(n):=\sum_{j \leqslant i} P_{j}(n)$. We twist the relative HN filtration (4.7) by $n$ and then pushforward under $\pi_{T}$ to obtain a filtration of $\pi_{T *}\left(\mathcal{U}_{T}(n)\right)$ by locally free sheaves $\pi_{T *}\left(\mathcal{U}_{T}^{(i)}(n)\right)$ of rank $P^{(i)}(n)$. Under the isomorphism (4.8), this induces a filtration of the trivial, rank $P(n)$, locally free sheaf $V_{n} \otimes \mathcal{O}_{T}$ over $T$ by vector subbundles of ranks $P^{(1)}(n)<P^{(2)}(n)<\cdots<P^{(s)}(n)=P(n)$, which corresponds to a unique morphism $h: T \rightarrow \operatorname{SL}\left(V_{n}\right) / P\left(V_{n}^{\bullet}\right)$ to the partial flag variety $\mathrm{SL}\left(V_{n}\right) / P\left(V_{n}^{\bullet}\right)$, where $P\left(V_{n}^{\bullet}\right) \subset \mathrm{SL}\left(V_{n}\right)$ is the parabolic subgroup associated to the filtration $V_{n}^{\bullet}$ given by $V_{n}^{(i)}=k^{P^{(i)}(n)}$. By the construction of the Hesselink index $\beta=([\lambda], d)$ associated to the HN type $\tau$, we have that $P(\lambda)=P\left(V_{n}^{\bullet}\right)$.

The pullback of the principal $P\left(V_{n}^{\bullet}\right)$-bundle

$$
\mathrm{SL}\left(V_{n}\right) \rightarrow \mathrm{SL}\left(V_{n}\right) / P\left(V_{n}^{\bullet}\right)
$$

to $T$ via $h$ is étale-locally trivial. In fact, it is Zariski-locally trivial as $P\left(V_{n}^{\bullet}\right)$ is a special group: the Levi factor is a product of general linear groups, which is special, and the unipotent factor is special as it is a chain of $\mathbb{G}_{a}$-extensions and the additive group $\mathbb{G}_{a}$ is special. Therefore, we have a Zariski cover $\left\{U_{l} \hookrightarrow T\right\}_{l \in L}$ such that the morphisms from $U_{l}$ to the flag variety can be lifted as shown in the following diagram:

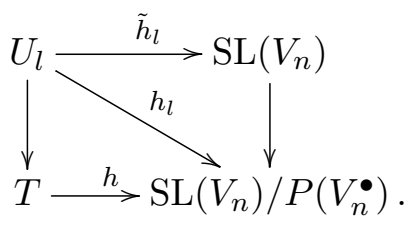

The morphism $\tilde{h}_{l}: U_{l} \rightarrow \mathrm{SL}\left(V_{n}\right)$ determines an isomorphism of sheaves

$$
V_{n} \otimes \mathcal{O}_{U_{l}} \rightarrow V_{n} \otimes \mathcal{O}_{U_{l}}
$$

and we let $g_{l}$ denote the induced isomorphism of the sheaf $V_{n} \otimes \pi_{X}^{*} \mathcal{O}_{X}(n)$ over $X \times U_{l}$. Let

$$
q_{l}: V_{n} \otimes \pi_{X}^{*} \mathcal{O}_{X}(n) \rightarrow \mathcal{U}_{l}
$$

denote the restriction of the surjection $q_{T}$ to $X \times U_{l}$; then the surjection $q_{l} \circ q_{l}^{-1}$ determines a morphism $s_{l}: U_{l} \rightarrow p_{\lambda}^{-1}\left(Z_{n, m}(\tau)\right)$, as the image of the filtration $V_{n}^{\bullet}$ under $q_{l} \circ q_{l}^{-1}$ gives the 


\section{Hoskins}

relative HN filtration of $\mathcal{U}_{l}$. Therefore, for each $l$, we have a morphism

$$
F_{l}: U_{l} \rightarrow \mathrm{SL}\left(V_{n}\right) \times p_{\lambda}^{-1}\left(Z_{n, m}(\tau)\right) \rightarrow \mathrm{SL}\left(V_{n}\right) \times{ }^{P(\lambda)} p_{\lambda}^{-1}\left(Z_{n, m}(\tau)\right) \rightarrow S_{\beta}^{\prime},
$$

where the first morphism is given by $\left(\tilde{h}_{l}, s_{l}\right)$ and the final morphism is given by the group action, which has image in $S_{\beta}^{\prime}$ as this is the smallest $G$-invariant subscheme of the Quot scheme which contains $p_{\lambda}^{-1}\left(Z_{n, m}(\tau)\right)$. By checking on double intersections, we see that the morphisms $F_{l}$ glue to give a morphism $F: T \rightarrow S_{\beta}^{\prime}$ (we note that the morphisms $\left(\tilde{h}_{l}, s_{l}\right)$ will not in general glue). This gives the required factorisation of $f: T \rightarrow Q_{n}^{o}$ and completes our proof.

The proof of the above theorem also proves the following corollary.

Corollary 4.20. Let $\tau \in \operatorname{HNT}(X, P)$; then for $m \gg n \gg 0$,

$$
Q_{n, \tau}=\mathrm{SL}\left(V_{n}\right) \cdot p_{\lambda}^{-1}\left(Z_{n, m}(\tau)\right),
$$

where $Z_{\tau, n, m}:=\prod_{i=1}^{s}\left(Q_{n}^{i}\right)^{\mathrm{ss}}$ and $\left(Q_{n}^{i}\right)^{\mathrm{ss}} \subset \operatorname{Quot}\left(V_{n}^{i} \otimes \mathcal{O}_{X}(-n), P_{i}\right)$ are the subschemes defined in the above proof.

Proposition 4.21. Let $\tau$ be a $H N$ type. Then, for $n \gg 0$, we have isomorphisms

$$
\mathcal{C o h}_{P}^{\tau}(X) \cong\left[Q_{n, \tau} / G_{n}\right]
$$

where $Q_{n, \tau}$ is a locally closed subscheme of $\operatorname{Quot}\left(V_{n} \otimes \mathcal{O}_{X}(-n), P\right)$ and $G_{n}=\operatorname{GL}\left(V_{n}\right)$.

Proof. By Corollary 4.20, there exists an $n \gg 0$ such that all sheaves with HN type $\tau$ are $n$-regular and so can be parametrised by a quotient sheaf in the HN stratum $Q_{n, \tau} \subset Q_{n}^{o}$.

The restriction $\mathcal{U}_{n, \tau}$ of the universal family to $Q_{n, \tau} \times X$ has the local universal property for families of sheaves on $X$ of HN type $\tau$ by our assumption on $n$. Therefore, we obtain a map

$$
Q_{n, \tau} \rightarrow \mathcal{C}^{\tau}{ }_{P}^{\tau}(X)
$$

that is an atlas for $\mathcal{C}_{P}^{\tau}(X)$. Two morphisms $f_{i}: S \rightarrow Q_{n, \tau}$ define isomorphic families of sheaves of HN type $\tau$ if and only if they are related by a morphism $\varphi: S \rightarrow \operatorname{GL}\left(V_{n}\right)$; that is, $f_{1}(s)=$ $\varphi(s) \cdot f_{2}(s)$ for all $s \in S$. Hence, the above morphism descends to an isomorphism $\left[Q_{n, \tau} / G_{n}\right] \rightarrow$ $\mathcal{C} \operatorname{ch}_{P}^{\tau}(X)$.

\subsection{Stratifications on the stack}

As explained in the introduction, one might naturally expect an agreement between the Hesselink and HN stratifications, following the work of Atiyah-Bott [AB83] and the agreement for quiver representations. However, Theorem 4.19 only gives a containment result. In this section, we explain why these stratifications do not agree and how to compare the stratifications on Quot $_{n}$ for different $n$ to produce an asymptotic stratification on the stack of coherent sheaves on $X$ which coincides with the HN stratification.

The following lemma indicates that we should work with the refined Hesselink strata $S_{\beta, \nu}$, where $\nu$ is a tuple of Hilbert polynomials (see Remark 4.8), rather than $S_{\beta}$; the proof of this lemma follows immediately from Definition 4.18.

Lemma 4.22. Let $\tau=\left(P_{1}, \ldots, P_{s}\right)$ and $\tau^{\prime}=\left(P_{1}^{\prime}, \ldots, P_{t}^{\prime}\right)$ be HN types; then $\beta_{n, m}(\tau)=\beta_{n, m}\left(\tau^{\prime}\right)$ if and only if $s=t$ and for $i=1, \ldots, s$, we have that $P_{i}(n)=P_{i}^{\prime}(n)$ and $P_{i}(m)=P_{i}^{\prime}(m)$.

Remark 4.23. We note that if $\operatorname{dim} X \leqslant 1$, then the assignment $\tau \mapsto \beta_{n, m}(\tau)$ is injective for any $m>n$, as the Hilbert polynomial $P_{i}$ of any sheaf on $X$ has at most degree 1 and so $P_{i}$ 


\section{STRATIFICATIONS IN MODULI PROBLEMS}

is uniquely determined by the pair $\left(P_{i}(n), P_{i}(m)\right)$. For $\operatorname{dim} X>1$, we cannot make the same conclusion. Although, for distinct HN types $\tau \neq \tau^{\prime}$, we note that

$$
\beta_{n, m}(\tau) \neq \beta_{n, m}\left(\tau^{\prime}\right) \quad \text { for } m \gg n \gg 0 .
$$

However, as there are infinitely many HN types, we cannot pick $m \gg n \gg 0$ such that the assignment $\tau \mapsto \beta_{n, m}(\tau)$ is injective for all HN types.

For each $n$ (and for each $m \gg n$ ), we have a refined Hesselink stratification of Quot ${ }_{n}$ associated to the linearisation $L_{n, m}$. If we fix $n$, then there are only finitely many Hesselink stratifications for the $\mathrm{SL}_{P(n)}$-action on Quot $_{n}$, by standard results on variation of GIT (see, for example, [DH98, Theorem 1.3.9]). Therefore, for $m_{n}$ sufficiently large, the Hesselink stratifications on Quot $_{n}$ associated to $L_{n, m}$ agree. We assume that we have taken $m_{n}$ sufficiently large that this is the case and will refer to the refined Hesselink stratification of Quot $_{n}$ with respect to $L_{n, m_{n}}$ as the refined Hesselink on Quot $_{n}$, which we write as follows:

$$
\operatorname{Quot}_{n}=\bigsqcup_{\beta \in \mathcal{B}_{n}, \nu \in \mathcal{C}_{\beta}} S_{\beta, \nu}^{n},
$$

where $\mathcal{B}_{n}$ is a finite set of conjugacy classes of rational 1-PSs of $\mathrm{SL}_{P(n)}$ and $\mathcal{C}_{\beta}$ is a finite set of tuples of Hilbert polynomials.

We now would like to allow $n$ to vary and study the limit of these stratifications as $n$ tends to infinity so that we can study all sheaves with Hilbert polynomials $P$ on $X$. However, there is one immediate problem: there are no natural morphisms Quot $_{n} \rightarrow$ Quot $_{n^{\prime}}$ for $n^{\prime}>n$ and so we cannot naturally produce an ind-scheme from these different Quot schemes. However, as every $n$-regular sheaf is $n^{\prime}$-regular for all $n^{\prime} \geqslant n$, it makes sense to restrict to an open subscheme $Q^{n \text {-reg }} \subset$ Quot $_{n}$ parametrising $n$-regular quotient sheaves $q: V_{n} \otimes \mathcal{O}_{X}(-n) \rightarrow \mathcal{E}$ such that $H^{0}(q(n))$ is an isomorphism. Then for $n^{\prime} \geqslant n$, we have natural equivariant morphisms

$$
Q^{n \text {-reg }} \rightarrow Q^{n^{\prime} \text {-reg }}
$$

Furthermore, the stack quotient of the $\mathrm{GL}_{P(n)}$-action on $Q^{n \text {-reg }}$ is isomorphic to the stack $\mathcal{C}_{P}{ }_{P}^{n \text {-reg }}(X)$ of $n$-regular coherent sheaves on $X$ with Hilbert polynomial $P$. Hence, the above morphisms determine a directed system of open immersions

$$
\mathcal{C o h}_{P}^{n \text {-reg }}(X) \hookrightarrow \mathcal{C}_{P}^{n^{\prime} \text {-reg }}(X)
$$

whose limit is the stack $\operatorname{Coh}_{P}(X)$ of coherent sheaves on $X$ with Hilbert polynomial $P$. This suggests that this stack is the correct space to compare the Hesselink stratifications for different $n$.

We can intersect the refined Hesselink stratification (4.9) with the open subscheme $Q^{n \text {-reg }}$ and then take the quotient by $G_{n}:=\mathrm{GL}_{P(n)}$ to obtain finite Hesselink stratifications

$$
\mathcal{C}^{n-\operatorname{reg}}(X)=\bigsqcup_{\beta \in \mathcal{B}_{n}, \nu \in \mathcal{C}_{\beta}} \mathcal{S}_{\beta, \nu}^{n},
$$

where the $\mathcal{S}_{\beta, \nu}^{n}$ are quotient stacks. More precisely, let $S_{\beta, \nu}^{n \text {-reg }}$ denote the fibre product of the

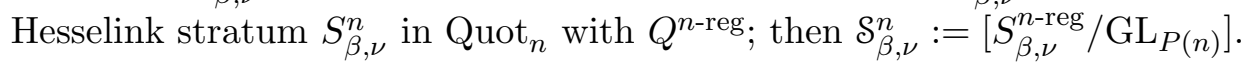

Definition 4.24. For each tuple $\nu$ of Hilbert polynomials, we let $\beta_{n}(\nu):=\beta_{n, m_{n}}(\nu) \in \mathcal{B}_{n}$ denote the associated Hesselink index given by Definition 4.18, and we write $\mathcal{S}_{\nu}^{n}:=\mathcal{S}_{\beta_{n}(\nu), \nu}^{n}$ for the corresponding refined Hesselink stratum in $\mathcal{C o h}_{P}^{n \text {-reg }}(X)$.

Proposition 4.25. Let $\nu=\left(P_{1}, \ldots, P_{s}\right)$ be a tuple of Hilbert polynomials on $X$ such that $\sum_{i} P_{i}=P$. 


\section{Hoskins}

(i) If $\nu=\tau \in \operatorname{HNT}(X, P)$, then for $n \gg 0$, we have a closed immersion of stacks

$$
\operatorname{Coh}_{P}^{\tau}(X) \hookrightarrow \mathcal{S}_{\tau}^{n}
$$

(ii) If $\nu=\tau \in \operatorname{HNT}(X, P)$ and $n \in \mathbb{N}$, then for $n^{\prime} \gg n$, we have a locally closed immersion

$$
\mathcal{S}_{\tau}^{n} \hookrightarrow \bigsqcup_{\tau^{\prime} \in \mathcal{B}_{\tau}^{n}} \mathcal{C}_{P}^{\tau^{\prime}}(X)
$$

where $\mathcal{B}_{\tau}^{n}$ is a finite set of $H N$ types $\tau^{\prime} \geqslant \tau$, and, moreover, for each $\tau^{\prime} \in \mathcal{B}_{\tau}^{n}$, we have a closed immersion $\mathcal{C o h}_{P}^{\tau^{\prime}}(X) \hookrightarrow \mathcal{S}_{\tau^{\prime}}^{n^{\prime}}$.

(iii) Let $\mathcal{F}$ be a sheaf on $X$ with Hilbert polynomial $P$. Then $\mathcal{F}$ is represented by a point in $\mathcal{S}_{\nu}^{n}$ for all $n \gg 0$ if and only if $\mathcal{F}$ has $H N$ type $\nu$ (and so, in particular, $\nu$ is a $H N$ type).

Proof. Part (i). By Theorem 4.19, for $n \gg 0$, the HN stratum $Q_{n, \tau}$ indexed by $\tau$ in the open subscheme $Q_{n}^{o} \subset$ Quot $_{n}$ is a closed subscheme of the refined Hesselink stratum $S_{\tau}^{n}:=S_{\beta_{n, m_{n}}(\tau), \tau}$ in Quot $_{n}$. The assumptions on $n$ given in Theorem 4.19 imply that all sheaves with HN type $\tau$ are $n$-regular, so the locally closed immersion $Q_{n, \tau} \hookrightarrow Q_{n}^{o}$ factors through the open subscheme $Q^{n \text {-reg }} \subset Q_{n}^{o}$. By the universal property of the fibre product, the closed immersion $Q_{n, \tau} \hookrightarrow S_{\tau}^{n}$ factors through $S_{\tau}^{n \text {-reg }}:=S_{\tau}^{n} \times{ }_{\operatorname{Quot}_{n}} Q^{n \text {-reg }}$. The factorisation $Q_{n, \tau} \hookrightarrow S_{\tau}^{n \text {-reg }}$ is a closed immersion (cf. [GD60, Corollaire 5.3.13]) and, after taking the stack quotient by $\mathrm{GL}_{P(n)}$, this induces a closed immersion $\mathcal{C}_{P}^{\tau}(X) \hookrightarrow \mathcal{S}_{\tau}^{n}$.

Part (ii). Since the refined Hesselink stratum $S_{\tau}^{n \text {-reg }}$ is a finite-type scheme, it has a finite stratification by HN types

$$
S_{\tau}^{n \text {-reg }}=\bigsqcup_{\tau^{\prime} \in \mathcal{B}_{\tau}^{n}} S_{\tau, \tau^{\prime}}^{n \text {-reg }},
$$

where $\mathcal{B}_{\tau}^{n}$ is a finite set of HN types. Furthermore, every quotient sheaf $q: V_{n} \otimes \mathcal{O}_{X}(-n) \rightarrow \mathcal{E}$ in $S_{\tau}^{n} \subset$ Quot $_{n}$ admits a filtration

$$
0 \subset \mathcal{E}^{(1)} \subset \mathcal{E}^{(2)} \subset \cdots \subset \mathcal{E}^{(s)}=\mathcal{E}
$$

such that $P\left(\mathcal{E}^{(i)} / \mathcal{E}^{(i-1)}\right)=P_{i}$; therefore, $\mathcal{E}$ has HN type at least $\tau$. Hence, for each $\tau^{\prime} \in \mathcal{B}_{\tau}^{n}$, we have $\tau^{\prime} \geqslant \tau$. As the set $\mathcal{B}_{\tau}^{n}$ is finite, we can apply part (i) to each HN type $\tau^{\prime} \in \mathcal{B}_{\tau}^{n}$ to conclude that for $n\left(\tau^{\prime}\right) \gg 0$, we have a closed immersion $\mathcal{C}_{0} \tau_{P}^{\tau^{\prime}}(X) \hookrightarrow \mathcal{S}_{\tau^{\prime}}^{n\left(\tau^{\prime}\right)}$. Let $n^{\prime}$ be the maximum of the $n\left(\tau^{\prime}\right)$ for $\tau^{\prime} \in \mathcal{B}_{\tau}^{n}$. Since $S_{\tau, \tau^{\prime}}^{n \text {-reg }} \subset S_{\tau}^{n \text {-reg }}$ is a locally closed subscheme, after taking the stack quotient by $\mathrm{GL}_{P(n)}$, we obtain a locally closed immersion. As a composition of immersions is an immersion, the composition

$$
\mathcal{S}_{\tau, \tau^{\prime}}^{n} \hookrightarrow \mathcal{S}_{\tau}^{n} \hookrightarrow \mathcal{C}_{\mathrm{oh}_{P}}^{n-\text { reg }}(X) \hookrightarrow \mathcal{C O h}^{n^{\prime} \text {-reg }}
$$

is a locally closed immersion, which factors via $\mathcal{C o h}_{P}^{\tau^{\prime}}(X)$ as $\mathcal{S}_{\tau, \tau^{\prime}}^{n}$ is the HN stratum for $\tau^{\prime}$ (to prove this, we use the natural atlases for these stacks and apply Theorem 4.15). The factorisation $\mathcal{S}_{\tau, \tau^{\prime}}^{n} \hookrightarrow \mathcal{C}_{\operatorname{oh}_{P}}^{\tau^{\prime}}(X)$ is then also a locally closed immersion. Hence, we have a locally closed immersion

$$
\mathcal{S}_{\tau}^{n}=\bigsqcup_{\tau^{\prime} \in \mathcal{B}_{\tau}^{n}} \mathcal{S}_{\tau, \tau^{\prime}}^{n} \hookrightarrow \bigsqcup_{\tau^{\prime} \in \mathcal{B}_{\tau}^{n}} \mathcal{C}_{P}^{\tau^{\prime}}(X)
$$

and, for each $\tau^{\prime} \in \mathcal{B}_{\tau}^{n}$, closed immersions $\mathcal{C}_{\operatorname{coh}}^{\tau^{\prime}}(X) \hookrightarrow \mathcal{S}_{\tau^{\prime}}^{n^{\prime}}$.

Part (iii). If $\mathcal{F}$ has HN type $\nu$, then $\mathcal{F}$ represents a point in $\mathcal{C o h}_{P}^{\nu}(X)$ and by part (i), this is contained in $\mathcal{S}_{\nu}^{n}$ for all $n \gg 0$. If $\mathcal{F}$ is represented by a point $\mathcal{S}_{\nu}^{n}$ for all $n \gg 0$ and has HN type $\tau \neq \nu$, then $\mathcal{F}$ represents a point in $\mathcal{C}_{\operatorname{coh}}^{\tau}(X)$ which is contained in $\mathcal{S}_{\tau}^{n}$ for all $n \gg 0$. This would contradict the fact that the refined Hesselink strata are disjoint. 


\section{STRATIFICATIONS IN MODULI PROBLEMS}

For a tuple $\nu$ of Hilbert polynomials, we have a refined Hesselink stratum $\mathcal{S}_{\nu}^{n} \subset \mathcal{C} \mathrm{oh}_{P}^{n \text {-reg }}(X)$ for each $n$. Unfortunately, it is not the case that for $n^{\prime} \gg n$, the $n^{\prime}$ th Hesselink stratification is a refinement of the $n$th Hesselink stratification. For example, for fixed $n$, we may have two $n$-regular sheaves $\mathcal{E}$ and $\mathcal{E}^{\prime}$ with the same HN type $\tau$, but the subquotients appearing in their HN filtrations are not necessarily $n$-regular. In this case, it is possible that $\mathcal{E}$ and $\mathcal{E}^{\prime}$ are represented by points in two distinct refined Hesselink strata $\mathcal{S}_{\nu}^{n}$ and $\mathcal{S}_{\nu^{\prime}}^{n}$, but for $n^{\prime} \gg n$, both sheaves will represent points in $\mathcal{S}_{\tau}^{n^{\prime}}$ by Proposition 4.25. However, for each $\nu$, from the collection of finite Hesselink strata $\mathcal{S}_{\nu}^{n}$ for different $n$, our aim is to construct an asymptotic 'limit' $\mathcal{S}_{\nu} \subset \mathcal{C} \mathcal{O h}_{P}(X)$ in the sense that a sheaf $\mathcal{F}$ is represented by a point of $\mathcal{S}_{\nu}$ if and only if $\mathcal{F}$ is represented by a point of $\mathcal{S}_{\nu}^{n}$ for $n \gg 0$.

A natural way to compare $S_{\nu}^{n}$ and $S_{\nu}^{n^{\prime}}$ for $n^{\prime}>n$ is to take their fibre product in $\mathcal{C o h}_{P}^{n^{\prime}-\text { reg }}(X)$. Definition 4.26. For a tuple of Hilbert polynomials and $n^{\prime}>n$, we let $\mathcal{S}_{\nu}^{n, n^{\prime}}$ be the following fibre product:

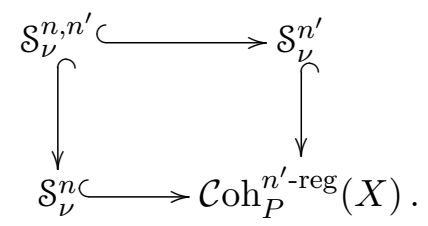

We note that a sheaf $\mathcal{E}$ is represented by a point in $\mathcal{S}_{\nu}^{n, n^{\prime}}$ if and only if it is $n$-regular and is represented by a point in $\mathcal{S}_{\nu}^{n}$ and a point in $\mathcal{S}_{\nu}^{n^{\prime}}$.

The following theorem shows that the fibre products $\mathcal{S}_{\nu}^{n, n^{\prime}}$ of the Hesselink strata stabilise for $n^{\prime} \gg n \gg 0$, and so we can define an asymptotic Hesselink stratum $\mathcal{S}_{\nu} \subset \mathcal{C o h}_{P}(X)$ to be this limit. Furthermore, we see that these asymptotic strata $\mathcal{S}_{\nu}$ give a stratification of $\mathcal{C o h}_{P}(X)$ which coincides with the Harder-Narasimhan stratification.

Theorem 4.27. Let $\nu=\left(P_{1}, \ldots, P_{s}\right)$ be a tuple of Hilbert polynomials on $X$ such that $\sum_{i} P_{i}=P$. For $n^{\prime} \geqslant n$, let $\mathcal{S}_{\nu}^{n, n^{\prime}}$ be the fibre product of the Hesselink strata $\mathcal{S}_{\nu}^{n}$ and $\mathcal{S}_{\nu}^{n^{\prime}}$ in $\mathcal{C}_{\mathrm{oh}}{ }_{P}^{n^{\prime}-\mathrm{reg}}(X)$.

(i) If $\nu \in \operatorname{HNT}(X, P)$, then for $n^{\prime} \gg n \gg 0$, we have $\mathcal{S}_{\nu}^{n, n^{\prime}}=\mathcal{C}_{\mathrm{Oh}_{P}^{\nu}}^{\nu}(X)$.

(ii) If $\nu \notin \operatorname{HNT}(X, P)$, then for $n^{\prime} \gg n \gg 0$, we have $\mathcal{S}_{\nu}^{n, n^{\prime}}=\emptyset$.

In either case, the refined Hesselink strata stabilise to an asymptotic stratum $\mathcal{S}_{\nu} \subset \mathcal{C o h}_{P}^{\nu}(X)$. Furthermore, the strata $\mathcal{S}_{\nu}$ give a stratification of $\mathcal{C}_{\mathrm{oh}}(X)$ which coincides with the HarderNarasimhan stratification.

Proof. Part (i). Take $n^{\prime} \gg n \gg 0$ as required by Proposition 4.25 such that we have immersions

$$
\mathcal{C o h}_{P}^{\nu}(X) \hookrightarrow \mathcal{S}_{\nu}^{n} \hookrightarrow \bigsqcup_{\nu^{\prime} \in \mathcal{B}_{\nu}^{n}} \mathcal{C o h}_{P}^{\nu^{\prime}}(X)
$$

for a finite set $\mathcal{B}_{\nu}^{n}$ of HN types $\nu^{\prime} \geqslant \nu$ and closed immersions $\mathcal{C o h}_{P}^{\nu^{\prime}}(X) \hookrightarrow \mathcal{S}_{\nu^{\prime}}^{n^{\prime}}$ for each $\nu^{\prime} \in \mathcal{B}_{\nu}^{n}$. By the construction of $\mathcal{S}_{\nu}^{n, n^{\prime}}$, we have locally closed immersions $\mathcal{S}_{\nu}^{n, n^{\prime}} \hookrightarrow \mathcal{S}_{\nu}^{n}$ and $\mathcal{S}_{\nu}^{n, n^{\prime}} \hookrightarrow \mathcal{S}_{\nu}^{n^{\prime}}$. Hence the composition

$$
\mathcal{S}_{\nu}^{n, n^{\prime}} \hookrightarrow \mathcal{S}_{\nu}^{n} \hookrightarrow \bigsqcup_{\nu^{\prime} \in \mathcal{B}_{\nu}^{n}} \mathcal{C o h}_{P}^{\nu^{\prime}}(X)
$$

is also a locally closed immersion. We claim that we have an immersion $\mathcal{S}_{\nu}^{n, n^{\prime}} \hookrightarrow \mathcal{C}_{P}{ }_{P}^{\nu}(X)$. Otherwise, $\mathcal{S}_{\nu}^{n, n^{\prime}}$ has non-empty intersection with $\mathcal{C o h}_{P}^{\nu^{\prime}}(X)$ for $\nu^{\prime} \neq \nu$, and this would contradict 


\section{Hoskins}

the disjointness of the (stack quotients of the refined) Hesselink strata $\mathcal{S}_{\nu}^{n^{\prime}}$ and $\mathcal{S}_{\nu^{\prime}}^{n^{\prime}}$. Since also $\mathcal{C} h_{P}^{\nu}(X) \hookrightarrow \mathcal{S}_{\nu}^{n}$ and $\mathcal{C o h}_{P}^{\nu}(X) \hookrightarrow \mathcal{S}_{\nu}^{n^{\prime}}$, and these maps are compatible with the maps to $\mathcal{C}{ }{ }_{P}(X)$, we conclude that $\mathcal{S}_{\nu}^{n, n^{\prime}}=\mathcal{C} \operatorname{oh}_{P}^{\nu}(X)$.

Part (ii). This follows directly from Proposition 4.25(iii) and the construction of $\mathcal{S}_{\nu}^{n, n^{\prime}}$.

In particular, we see that an asymptotic Hesselink stratum $\mathcal{S}_{\nu}$ is non-empty if and only if $\nu \in \operatorname{HNT}(X, P)$, and these asymptotic Hesselink strata coincide with the HN strata.

Remark 4.28. A sheaf is represented by a point of $\mathcal{S}_{\nu}$ if and only if it is represented by a point of $\mathcal{S}_{\nu}^{n}$ for $n \gg 0$. To prove this claim, we note that a sheaf $\mathcal{F}$ has HN type $\nu$ if and only if it represents a point in $\mathcal{S}_{n}^{\nu}$ for $n \gg 0$ by Proposition 4.25 (iii).

\section{A functor from sheaves to quiver representations}

Álvarez-Cónsul and King give a functorial construction of moduli spaces of semistable sheaves on $\left(X, \mathcal{O}_{X}(1)\right)$ using moduli spaces of representations for a Kronecker quiver [AK07]. In this section, we generalise this construction by extending this quiver and compare the HN strata for sheaves and quivers.

\subsection{Overview of the construction of the functor}

Let $X$ be a projective scheme of finite type over $k$ with very ample line bundle $\mathcal{O}_{X}(1)$, and let $\operatorname{Coh}(X)$ denote the category of coherent sheaves on $X$. For natural numbers $m>n$, we let $K_{n, m}$ be a Kronecker quiver with vertex set $V:=\{n, m\}$ and $\operatorname{dim} H^{0}\left(\mathcal{O}_{X}(m-n)\right)$ arrows from $n$ to $m$ and consider the functor

$$
\Phi_{n, m}:=\operatorname{Hom}\left(\mathcal{O}_{X}(-n) \oplus \mathcal{O}_{X}(-m),-\right): \operatorname{Coh}(X) \rightarrow \operatorname{Rep}\left(K_{n, m}\right)
$$

that sends a sheaf $\mathcal{E}$ to the representation $W_{\mathcal{E}}$ of $K_{n, m}$, where

$$
W_{\mathcal{E}, n}:=H^{0}(\mathcal{E}(n)), \quad W_{\mathcal{E}, m}:=H^{0}(\mathcal{E}(m))
$$

and the evaluation map $H^{0}(\mathcal{E}(n)) \otimes H^{0}\left(\mathcal{O}_{X}(m-n)\right) \rightarrow H^{0}(\mathcal{E}(m))$ gives the morphisms.

Let $\mathbf{C o h}_{P}^{n \text {-reg }}(X)$ be the subcategory of $\mathbf{C o h}(X)$ consisting of $n$-regular sheaves with Hilbert polynomial $P$. Then the image of $\Phi_{n, m}$ restricted to $\operatorname{Coh}_{P}^{n \text {-reg }}(X)$ is contained in the subcategory of quiver representations of dimension vector $d_{n, m}(P)=(P(n), P(m))$. By [AK07, Theorem 3.4], if $\mathcal{O}_{X}(m-n)$ is regular, then the functor

$$
\Phi_{n, m}: \operatorname{Coh}_{P}^{n-\operatorname{reg}}(X) \rightarrow \operatorname{Rep}_{d_{n, m}(P)}\left(K_{n, m}\right)
$$

is fully faithful. For representations of $K_{n, m}$ of dimension $d_{n, m}(P)$, we consider the stability parameter $\theta_{n, m}(P):=(-P(m), P(n))$.

Theorem 5.1 ([AK07, Theorem 5.10]). For $m \gg n \gg 0$, depending on $X$ and $P$, a sheaf $\mathcal{E}$ with Hilbert polynomial $P$ is semistable if and only if it is pure and n-regular and the quiver representation $\Phi_{n, m}(\mathcal{E})$ is $\theta_{n, m}(P)$-semistable.

We briefly describe how to pick $m \gg n \gg 0$ as required for this theorem to hold; for further details, we refer to the reader to the conditions (C:1)-(C:5) stated in [AK07, §5.1]. First, we take $n$ such that all semistable sheaves with Hilbert polynomial $P$ are $n$-regular and the Le Potier-Simpson estimates hold. Then, we choose $m$ such that $\mathcal{O}_{X}(m-n)$ is regular and for all $n$-regular sheaves $\mathcal{E}$ and vector subspaces $V^{\prime} \subset H^{0}(\mathcal{E}(n))$, the subsheaf $\mathcal{E}^{\prime}$ generated by $V^{\prime}$ under 


\section{STRATIFICATIONS IN MODULI PROBLEMS}

the evaluation map $H^{0}(\mathcal{E}(n)) \otimes \mathcal{O}(-n) \rightarrow \mathcal{E}$ is $m$-regular. Finally, we take $m$ sufficiently large that a finite list of polynomial inequalities can be determined by evaluation at $m$ (see (C:5) in $[\mathrm{AK} 07])$.

We can alternatively consider the functor $\Phi_{n, m}$ as a morphism of stacks. We recall that

$$
\mathcal{C}_{P}^{n-\text { reg }}(X) \cong\left[Q^{n-\text { reg }} / G_{n}\right]
$$

where $G_{n}=\operatorname{GL}\left(V_{n}\right)$, and

$$
\mathcal{R e p}_{d_{n, m}(P)}\left(K_{n, m}\right) \cong\left[\operatorname{Rep}_{d_{n, m}(P)}\left(K_{n, m}\right) / \bar{G}_{d_{n, m}(P)}\left(K_{n, m}\right)\right] .
$$

Let $\mathcal{U}_{n}$ be the universal quotient sheaf over $Q^{n \text {-reg }} \times X$, and let $p: Q^{n \text {-reg }} \times X \rightarrow Q^{n \text {-reg }}$ be the projection map. By the definition of $Q^{n \text {-reg }}$, we have that $R^{i} p_{*}\left(\mathcal{U}_{n}(n)\right)=0$ for $i>0$; therefore, by the semi-continuity theorem, $p_{*}\left(\mathcal{U}_{n}(n)\right)$ is a vector bundle over $Q^{n \text {-reg }}$ of rank $P(n)$, and similarly $p_{*}\left(\mathcal{U}_{n}(m)\right)$ is a rank $P(m)$ vector bundle. Hence, by using the evaluation map, we obtain a family



$$
Q^{n-\mathrm{reg}} \rightarrow \mathcal{R e p}_{d_{n, m}(P)}\left(K_{n, m}\right) .
$$

As this morphism is $G_{n}$-invariant, it descends to a morphism of stacks

$$
\Phi_{n, m}: \mathcal{C}_{P}^{n-r e g}(X) \rightarrow \mathcal{R e p}_{d_{n, m}(P)}\left(K_{n, m}\right) .
$$

We study the images of Hesselink (respectively, HN) strata under this morphism in Proposition 5.5 (respectively, Theorem 5.7) below.

\subsection{The images of the Hesselink strata}

In this section, we fix $n$ and assume $m \gg n$ such that

(A1) $\mathcal{O}_{X}(m-n)$ is regular,

(A2) for any $n$-regular sheaf $\mathcal{E}$ and any vector subspace $V^{\prime} \subset H^{0}(\mathcal{E}(n))$, the subsheaf $\mathcal{E}^{\prime} \subset \mathcal{E}$ generated by $V^{\prime}$ is $m$-regular.

The second assumption is possible as such sheaves $\mathcal{E}^{\prime}$ form a bounded family (see [AK07, Condition (C:4) in $\S 5.1])$.

Let

$$
\operatorname{Quot}\left(V_{n} \otimes \mathcal{O}_{X}(-n), P\right)=\bigsqcup_{\beta \in \mathcal{B}_{n, m}} S_{\beta}
$$

be the Hesselink stratification associated to the $\mathrm{SL}\left(V_{n}\right)$-action on this Quot scheme with respect to $L_{n, m}$ as we described in Section 4.3. As in Section 4.7, we consider the induced stratification on the stack of $n$-regular sheaves

$$
\mathcal{C o h}_{P}^{n-\text { reg }}(X)=\bigsqcup_{\beta} \mathcal{S}_{\beta}^{n, m}
$$

where $\mathcal{S}_{\beta}^{n, m}=\left[S_{\beta}^{n \text {-reg }} / G_{n}\right]$ and $S_{\beta}^{n \text {-reg }}$ is the fibre product of $Q^{n \text {-reg }}$ and $S_{\beta}$ in this Quot scheme.

To define a Hesselink stratification on the space (or stack) of representations of $K_{n, m}$ of dimension vector $d_{n, m}(P)$, we need to choose a parameter $\alpha \in \mathbb{N}^{2}$ which defines a norm $\|-\|_{\alpha}$ as in Example 2.4(b). We take $\alpha=\alpha_{n, m}(P):=(P(m), P(n))$ due to the following lemma.

Lemma 5.2. Let $\theta=\theta_{n, m}(P)$ and $\alpha=\alpha_{n, m}(P)$. Then, for sheaves $\mathcal{E}$ and $\mathcal{F}$, we have

$$
\frac{\theta\left(W_{\mathcal{E}}\right)}{\alpha\left(W_{\mathcal{E}}\right)} \geqslant \frac{\theta\left(W_{\mathcal{F}}\right)}{\alpha\left(W_{\mathcal{F}}\right)} \Longleftrightarrow \frac{h^{0}(\mathcal{E}(n))}{h^{0}(\mathcal{E}(m))} \leqslant \frac{h^{0}(\mathcal{F}(n))}{h^{0}(\mathcal{F}(m))} .
$$




\section{Hoskins}

The same statement holds if we replace these inequalities with strict inequalities.

Proof. By the definitions of these parameters, we have

$$
\frac{\theta\left(W_{\mathcal{E}}\right)}{\alpha\left(W_{\mathcal{E}}\right)}:=\frac{-P(m) h^{0}(\mathcal{E}(n))+P(n) h^{0}(\mathcal{E}(m))}{P(m) h^{0}(\mathcal{E}(n))+P(n) h^{0}(\mathcal{E}(m))}=1-\frac{2 P(m) h^{0}(\mathcal{E}(n))}{P(m) h^{0}(\mathcal{E}(n))+P(n) h^{0}(\mathcal{E}(m))} .
$$

From this, it is easy to check the desired equivalences of inequalities.

By Theorem 3.8, we can equivalently view the Hesselink stratification (with respect to $\rho_{\theta}$ and $\|-\|_{\alpha}$ ) as a stratification by $\mathrm{HN}$ types (with respect to $\theta$ and $\alpha$ ):

$$
\mathcal{R e p}_{d_{n, m}(P)}\left(K_{n, m}\right)=\bigsqcup_{\gamma} \mathcal{R e p}_{d_{n, m}(P)}^{\gamma}\left(K_{n, m}\right)
$$

The unstable Hesselink strata in (5.1) are indexed by conjugacy classes of rational 1-PSs $\lambda_{\beta}$ of $\operatorname{SL}\left(V_{n}\right)$. Equivalently, the index $\beta$ is given by a collection of strictly decreasing rational weights $r(\beta)=\left(r_{1}, \ldots, r_{s}\right)$ and multiplicities $l(\beta)=\left(l_{1}, \ldots, l_{s}\right)$ satisfying $\sum_{i=1}^{s} l_{i}=P(n)$ and $\sum_{i=1}^{s} r_{i} l_{i}=0$. More precisely, we recall that the rational 1-PS associated to $r(\beta)$ and $l(\beta)$ is

$$
\lambda_{\beta}(t)=\operatorname{diag}\left(t^{r_{1}}, \ldots, t^{r_{1}}, \ldots, t^{r_{s}}, \ldots, t^{r_{s}}\right),
$$

where $r_{i}$ appears $l_{i}$ times.

Definition 5.3. For an index $\beta$ of the Hesselink stratification (5.1) on the stack of $n$-regular sheaves as above, we let $\gamma(\beta):=\left(d_{1}(\beta), \ldots, d_{s}(\beta)\right)$, where

$$
d_{i}(\beta)=\left(l_{i}, l_{i} \frac{P(m)}{P(n)}-l_{i} r_{i}\right) .
$$

Lemma 5.4. Let $\beta$ be an index for the Hesselink stratification (5.1); then $\gamma(\beta)$ is a HN type for a representation of $K_{n, m}$ of dimension $d_{n, m}$ with respect to $\theta$ and $\alpha$.

Proof. Let $r(\beta)=\left(r_{1}, \ldots, r_{s}\right)$ and $l(\beta)=\left(l_{1}, \ldots, l_{s}\right)$ be as above. Then

$$
\sum_{i=1}^{s} d_{i}(\beta)=\left(\sum_{i=1}^{n} l_{i}, \sum_{i=1}^{n} l_{i} \frac{P(m)}{P(n)}-l_{i} r_{i}\right)=(P(n), P(m))
$$

as $\sum l_{i}=P(n)$ and $\sum l_{i} r_{i}=0$. Furthermore, as $r_{1}>\cdots>r_{s}$, it follows that

$$
\frac{\theta\left(d_{1}(\beta)\right)}{\alpha\left(d_{1}(\beta)\right)}<\frac{\theta\left(d_{2}(\beta)\right)}{\alpha\left(d_{2}(\beta)\right)}<\cdots<\frac{\theta\left(d_{s}(\beta)\right)}{\alpha\left(d_{s}(\beta)\right)} .
$$

To complete the proof, we must verify that

$$
d_{i}(\beta)=\left(l_{i}, l_{i} \frac{P(m)}{P(n)}-l_{i} r_{i}\right) \in \mathbb{N}^{2} .
$$

The first number $l_{i}$ is a multiplicity and so is a natural number, but the second number is a priori only rational. As $\beta$ is an index for the Hesselink stratification, it indexes a non-empty stratum $S_{\beta}$ and, as this stratum is constructed from its associated limit set $Z_{d}^{\lambda}$, by Theorem 2.8, it follows that this limit set must also be non-empty. Hence, this limit set contains a quotient sheaf $q: V_{n} \otimes \mathcal{O}_{X}(-n) \rightarrow \oplus_{i=1}^{s} \mathcal{E}_{i}$ such that, by Proposition 4.6, for $i=1, \ldots, s$, we have

$$
P\left(\mathcal{E}_{i}, m\right)=l_{i} \frac{P(m)}{P(n)}-l_{i} r_{i} .
$$

As $P\left(\mathcal{E}_{i}, m\right) \in \mathbb{N}$, this completes the proof. 


\section{STRATIFICATIONS IN MODULI PROBLEMS}

Proposition 5.5. For a Hesselink index $\beta$ in (5.1), we have

$$
\Phi_{n, m}\left(\mathcal{S}_{\beta}^{n, m}\right) \subset \bigsqcup_{\gamma \geqslant \gamma(\beta)} \mathcal{R e p}_{d_{n, m}(P)}^{\gamma}\left(K_{n, m}\right) .
$$


$l(\beta)=\left(l_{1}, \ldots, l_{s}\right)$ be the associated rational weights and multiplicities; thus, $r_{1}>\cdots>r_{s}$ and

$$
\lambda_{\beta}(t)=\operatorname{diag}\left(t^{r_{1}}, \ldots, t^{r_{1}}, \ldots, t^{r_{s}}, \ldots, t^{r_{s}}\right),
$$

where $r_{i}$ appears $l_{i}$ times. If $\lambda$ denotes the unique integral primitive 1-PS associated to $\lambda_{\beta}$, then $\beta=([\lambda], d)$, where $d=\left\|\lambda_{\beta}\right\|$. The 1-PS $\lambda$ induces a filtration $0=V_{n}^{(0)} \subset V_{n}^{(1)} \subset \cdots \subset V_{n}^{(s)}=V_{n}$ such that the successive quotients $V_{n}^{i}$ have dimensions $l_{i}$.

By Corollary 4.7, there exists a $g \in \mathrm{SL}\left(V_{n}\right)$ such that we have a filtration

$$
0=\mathcal{E}^{(0)} \subset \cdots \subset \mathcal{E}^{(i)}:=g \cdot q\left(V_{n}^{(i)} \otimes \mathcal{O}_{X}(-n)\right) \subset \cdots \subset \mathcal{E}^{(s)}=\mathcal{E},
$$

where the Hilbert polynomials of $\mathcal{E}^{i}:=\mathcal{E}^{(i)} / \mathcal{E}^{(i-1)}$ satisfy

$$
P(n) P\left(\mathcal{E}_{i}, m\right)=l_{i}\left(P(m)-r_{i} P(n)\right) \text { for } i=1, \ldots, s .
$$

Let $W^{(i)}:=\Phi_{n, m}\left(\mathcal{E}^{(i)}\right)$; then we have a filtration

$$
0=W^{(0)} \subset W^{(1)} \subset \cdots \subset W^{(s)}=W
$$

with $\operatorname{dim} W^{(i)}:=\left(\operatorname{dim} H^{0}\left(\mathcal{E}^{(i)}(n)\right), \operatorname{dim} H^{0}\left(\mathcal{E}^{(i)}(m)\right)\right)=\left(\operatorname{dim} V_{n}^{(i)}, P\left(\mathcal{E}^{(i)}, m\right)\right)$, due to the fact that $H^{0}(q(n))$ is an isomorphism (because $q \in Q^{n \text {-reg }}$ ) and $\mathcal{E}^{(i)}$ is $m$-regular (by the assumption $(\mathrm{A} 2)$ on $m$ ). Let $W_{i}:=W^{(i)} / W^{(i-1)}$; then

$$
\operatorname{dim} W_{i}=\left(\operatorname{dim} V_{n}^{i}, P\left(\mathcal{E}_{i}, m\right)\right)=\left(l_{i}, l_{i} \frac{P(m)}{P(n)}-l_{i} r_{i}\right) .
$$

As we have a filtration (5.2) of $W$ whose successive quotients have dimension vectors specified by $\gamma(\beta)$ and $\gamma(\beta)$ is a HN type for $d_{n, m}$-dimensional representation of $K_{n, m}$ by Lemma 5.4 , it follows that $W=\Phi_{n, m}(\mathcal{E})$ has $\mathrm{HN}$ type greater than or equal to $\gamma(\beta)$.

In the above proof, we note that the subsheaves $\mathcal{E}^{(i)}$ may not be $n$-regular and so $W_{i}$ is only isomorphic to $\Phi_{n, m}\left(\mathcal{E}_{i}\right)$ if $H^{1}\left(\mathcal{E}^{(i-1)}(n)\right)=0$. This is not the case in general, although $W_{i}$ is always a subrepresentation of $\Phi_{n, m}\left(\mathcal{E}_{i}\right)$. Hence, it is not possible to use GIT semistability properties of the quotients $q_{i}: V_{n}^{i} \otimes \mathcal{O}_{X}(-n) \rightarrow \mathcal{E}_{i}$ to deduce the $(\theta, \alpha)$-semistability of $W_{i}$ (that is, to show that (5.2) is the HN filtration of $W$ ).

\subsection{The images of the HN strata}

In this section, we study the image of the $\mathrm{HN}$ strata $\mathcal{C o h}_{P}^{\tau}(X)$ under the map

$$
\Phi_{n, m}: \mathcal{C o h}_{P}^{n \text {-reg }}(X) \rightarrow \mathcal{R e p}_{d_{n, m}(P)}\left(K_{n, m}\right)
$$

for $n$ and $m$ sufficiently large (depending on $\tau$ ). We prove that a HN stratum for sheaves is mapped to a HN stratum for quiver representations. Let $\theta=\theta_{n, m}(P):=(-P(m), P(n))$ and $\alpha=\alpha_{n, m}(P):=(P(m), P(n))$ be as above.

Definition 5.6. For a $\operatorname{HN}$ type $\tau=\left(P_{1}, \ldots, P_{s}\right) \in \operatorname{HNT}(X, P)$ and $(n, m) \in \mathbb{N}^{2}$, we let

$$
\gamma_{n, m}(\tau):=\left(d_{n, m}\left(P_{1}\right), \ldots, d_{n, m}\left(P_{s}\right)\right),
$$

where $d_{n, m}\left(P_{i}\right)=\left(P_{i}(n), P_{i}(m)\right)$. 


\section{Hoskins}

As $\tau$ is a HN type of sheaves, we have that $P_{1} \succ P_{2} \succ \cdots \succ P_{s}$; thus,

$$
\frac{P_{1}(n)}{P_{1}(m)}>\frac{P_{2}(n)}{P_{2}(m)}>\cdots>\frac{P_{s}(n)}{P_{s}(m)} \quad \text { for } m \gg n \gg 0 .
$$

Therefore, by Lemma 5.2, for $m \gg n \gg 0$, we have

$$
\frac{\theta\left(d_{n, m}\left(P_{1}\right)\right)}{\alpha\left(d_{n, m}\left(P_{1}\right)\right)}<\frac{\theta\left(d_{n, m}\left(P_{2}\right)\right)}{\alpha\left(d_{n, m}\left(P_{2}\right)\right)}<\cdots<\frac{\theta\left(d_{n, m}\left(P_{s}\right)\right)}{\alpha\left(d_{n, m}\left(P_{s}\right)\right)}
$$

that is, $\gamma_{n, m}(\tau)$ is a HN type for representations of $K_{n, m}$ of dimension $d_{n, m}(P)$ for $m \gg n \gg 0$.

Theorem 5.7. Let $\tau=\left(P_{1}, \ldots, P_{s}\right) \in \operatorname{HNT}(X, P)$ be a $H N$ type. Then, for $m \gg n \gg 0$,

$$
\Phi_{n, m}\left(\mathcal{C o h}_{P}^{\tau}(X)\right) \subset \mathcal{R} \operatorname{ep}_{d_{n, m}(P)}^{\gamma_{n, m}(\tau)}\left(K_{n, m}\right) .
$$

Proof. We take $m \gg n \gg 0$ as needed for Theorem 5.1 for the Hilbert polynomials $P_{1}, \ldots, P_{s}$. Furthermore, we assume that $m$ and $n$ are sufficiently large that the inequalities (5.3) hold.

Let $\mathcal{E}$ be a sheaf on $X$ of HN type $\tau$ and HN filtration given by

$$
0=\mathcal{E}^{(0)} \subset \mathcal{E}^{(1)} \subset \cdots \subset \mathcal{E}^{(s)}=\mathcal{E} .
$$

Let $W=\Phi_{n, m}(\mathcal{E})$ and $W^{(i)}:=\Phi_{n, m}\left(\mathcal{E}^{(i)}\right)$; then we claim that the induced filtration

$$
0=W^{(0)} \subset W^{(1)} \subset \cdots \subset W^{(s)}=W
$$

is the $\mathrm{HN}$ filtration of $W$ with respect to $(\theta, \alpha)$ and, moreover, that $W$ has HN type $\gamma_{n, m}(\tau)$. Let $\mathcal{E}_{i}$ and $W_{i}$ denote the successive subquotients in the above filtrations. Our assumptions on $n$ imply that each $\mathcal{E}_{i}$ is $n$-regular and so, by induction, each $\mathcal{E}^{(i)}$ is $n$-regular. Therefore, we have exact sequences

$$
0 \rightarrow H^{0}\left(\mathcal{E}^{(i-1)}(n)\right) \rightarrow H^{0}\left(\mathcal{E}^{(i)}(n)\right) \rightarrow H^{0}\left(\mathcal{E}_{i}(n)\right) \rightarrow 0
$$

that give isomorphisms $\Phi_{n, m}\left(\mathcal{E}_{i}\right) \cong W_{i}$. Since $\mathcal{E}_{i}$ is $n$-regular and semistable with Hilbert polynomial $P_{i}$, the quiver representation $W_{i}$ is $\theta_{n, m}\left(P_{i}\right)$-semistable by Theorem 5.1. Set $\theta_{i}:=\theta_{n, m}\left(P_{i}\right)$. For a subrepresentation $W^{\prime} \subset W_{i}$, we note that

$$
\theta_{i}\left(W^{\prime}\right) \geqslant 0 \Longleftrightarrow P_{i}(n) \operatorname{dim} W_{v_{m}}^{\prime} \geqslant P_{i}(m) \operatorname{dim} W_{v_{n}}^{\prime} \Longleftrightarrow \frac{\theta\left(W^{\prime}\right)}{\alpha\left(W^{\prime}\right)} \geqslant \frac{\theta\left(W_{i}\right)}{\alpha\left(W_{i}\right)} .
$$

Therefore, $\theta_{i}$-semistability of $W_{i}$ implies $(\theta, \alpha)$-semistability of $W_{i}$. To finish the proof of the claim, that is, to prove that (5.4) is the HN filtration of $W$, it suffices to check that

$$
\frac{\theta\left(W_{1}\right)}{\alpha\left(W_{1}\right)}<\frac{\theta\left(W_{2}\right)}{\alpha\left(W_{2}\right)}<\cdots<\frac{\theta\left(W_{s}\right)}{\alpha\left(W_{s}\right)}
$$

or, equivalently, by Lemma 5.2, that

$$
\frac{h^{0}\left(\mathcal{E}_{1}(n)\right)}{h^{0}\left(\mathcal{E}_{1}(m)\right)}>\frac{h^{0}\left(\mathcal{E}_{2}(n)\right)}{h^{0}\left(\mathcal{E}_{2}(m)\right)}>\cdots>\frac{h^{0}\left(\mathcal{E}_{s}(n)\right)}{h^{0}\left(\mathcal{E}_{s}(m)\right)} .
$$

Since $\mathcal{E}_{i}$ is $n$-regular, we have that $h^{0}\left(\mathcal{E}_{i}(n)\right)=P_{i}(n)$ and $h^{0}\left(\mathcal{E}_{i}(m)\right)=P_{i}(m)$; hence, the above inequalities are equivalent to (5.3). Moreover, this shows that $W$ has $\mathrm{HN}$ type $\gamma_{n, m}(\tau)$.

Remark 5.8. The assignment $\tau \mapsto \gamma_{n, m}(\tau)$ is not injective for exactly the same reason as in Lemma 4.22. In fact, more generally, $P \mapsto d_{n, m}(P)$ is not injective unless $\operatorname{dim} X \leqslant 1$. 


\section{STRATIFICATIONS IN MODULI PROBLEMS}

\subsection{Adding more vertices}

In this final section, we generalise the construction of Álvarez-Cónsul and King by adding more vertices so that the map $\tau \mapsto \gamma(\tau)$ is injective.

For a tuple $\underline{n}=\left(n_{0}, \ldots, n_{d}\right)$ of increasing natural numbers, we define a functor

$$
\Phi_{\underline{n}}:=\operatorname{Hom}\left(\bigoplus_{i=0}^{d} \mathcal{O}_{X}\left(-n_{i}\right),-\right): \mathcal{C} \operatorname{oh}(X) \rightarrow \mathcal{R} \operatorname{ep}\left(K_{\underline{n}}\right),
$$

where $K_{\underline{n}}$ denotes the quiver with vertex set $V=\left\{n_{0}, \ldots, n_{d}\right\}$ and $\operatorname{dim} H^{0}\left(\mathcal{O}_{X}\left(n_{i+1}-n_{i}\right)\right)$ arrows from $n_{i}$ to $n_{i+1}$ :

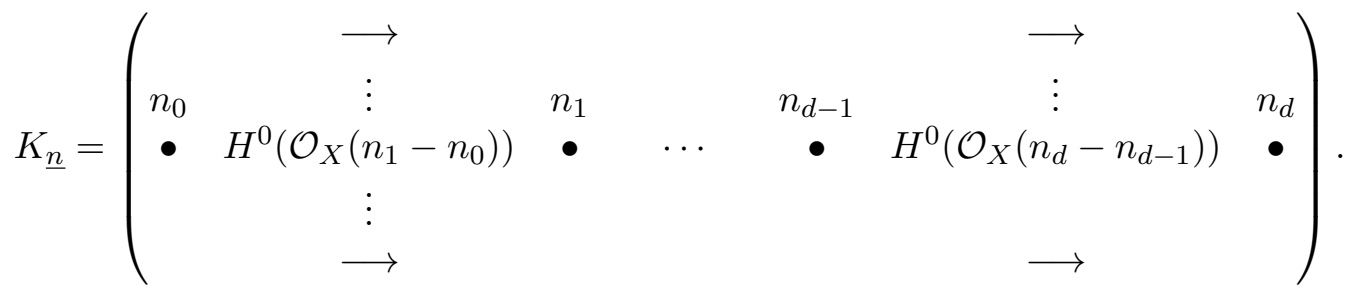

More precisely, if $\mathcal{E}$ is a coherent sheaf on $X$, then $\Phi_{n}(\mathcal{E})$ is the quiver representation denoted by $W_{\mathcal{E}}=\left(W_{\mathcal{E}, n_{0}}, \ldots, W_{\mathcal{E}, n_{d}}, \operatorname{ev}_{\mathcal{E}}^{1}, \ldots\right.$, ev $\left._{\mathcal{E}}^{d}\right)$, where we let $W_{\mathcal{E}, i}:=H^{0}(\mathcal{E}(i))$ and the arrows are given by the evaluation maps ev $\mathcal{E}_{\mathcal{E}}^{i}: H^{0}\left(\mathcal{E}\left(n_{i-1}\right)\right) \otimes H^{0}\left(\mathcal{O}\left(n_{i}-n_{i-1}\right)\right) \rightarrow H^{0}\left(\mathcal{E}\left(n_{i}\right)\right)$.

We note that $\Phi_{\underline{n}}$ maps $n_{0}$-regular sheaves with Hilbert polynomial $P$ to representations of $K_{\underline{n}}$ of dimension vector $d_{\underline{n}}(P):=\left(P\left(n_{0}\right), \ldots, P\left(n_{d}\right)\right)$.

Remark 5.9. In general, for $d>1$, the image of $\Phi_{\underline{n}}$ is contained in a substack consisting of quiver representations satisfying certain relations $\mathcal{R}_{\underline{n}}$. To describe the relations, we consider the evaluation maps $\operatorname{ev}_{\mathcal{O}}^{i}: H^{0}\left(\mathcal{O}_{X}\left(n_{i}-n_{i-1}\right)\right) \otimes H^{0}\left(\mathcal{O}_{X}\left(n_{i+1}-n_{i}\right)\right) \rightarrow H^{0}\left(\mathcal{O}_{X}\left(n_{i+1}-n_{i-1}\right)\right)$. If $H_{i}:=H^{0}\left(\mathcal{O}_{X}\left(n_{i}-n_{i-1}\right)\right)$, we have commutative squares

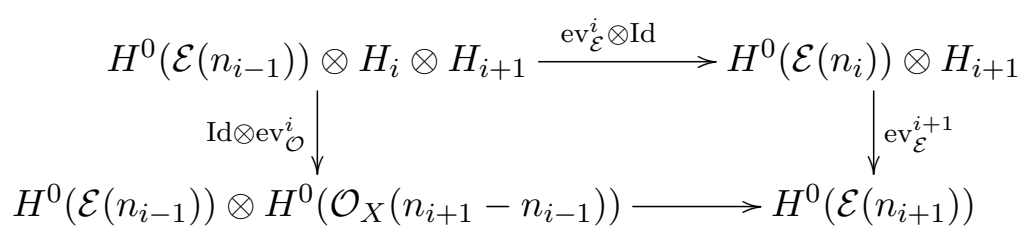

and, therefore, elements in the kernel of $\mathrm{ev}_{\mathcal{O}, i}$ give rise to compositions of paths that are zero. More precisely, the kernels of the iterated evaluation maps

$$
H_{i} \otimes H_{i+1} \otimes \cdots \otimes H_{j} \rightarrow H^{0}\left(\mathcal{O}_{X}\left(n_{j}-n_{i-1}\right)\right),
$$

for $i<j$, describe the set of relations $\mathcal{R}_{\underline{n}}$ on $K_{\underline{\underline{n}}}$.

Remark 5.10. In this remark, we explain how many constructions of [AK07] can be generalised. Throughout, we assume that the $\mathcal{O}_{X}\left(n_{i}-n_{i-1}\right)$ are 0 -regular for $i=1, \ldots, d$, so that

$$
\text { ev: } H^{0}\left(\mathcal{O}_{X}\left(n_{i}-n_{i-1}\right)\right) \otimes H^{0}\left(\mathcal{O}_{X}(l)\right) \rightarrow H^{0}\left(\mathcal{O}_{X}\left(n_{i}-n_{i-1}+l\right)\right)
$$

is surjective for $l \geqslant 0$. Let $B_{\underline{n}}$ be the path algebra of $K_{\underline{n}}$. Then the category of representations of $K_{\underline{n}}$ is equivalent to the category of finite-dimensional right $B_{\underline{\underline{n}}}$-modules. As a vector space,

$$
B_{\underline{n}}=\bigoplus_{i=0}^{d} k e_{i} \oplus \bigoplus_{i=1}^{d} H_{i} \oplus \bigoplus_{i=2}^{d} H_{i-1} \otimes H_{i} \quad \oplus \cdots \oplus H_{1} \otimes H_{2} \otimes \cdots \otimes H_{d},
$$

$$
\text { path length: } \quad 0 \quad 1 \quad 2 \quad \ldots \quad d
$$

where $e_{i}$ is the trivial path at vertex $i$ and $H_{i}:=H^{0}\left(\mathcal{O}_{X}\left(n_{i}-n_{i-1}\right)\right)$ is the space of arrows $i-1 \mapsto i$. Let $L_{\underline{n}}$ and $H_{\underline{n}}$ denote the spaces of length 0 and length 1 paths, respectively; 


\section{Hoskins}

then $H_{\underline{n}}$ is an $L_{\underline{n}}$-bimodule, with structure characterised by $e_{i-1} H_{\underline{n}} e_{i}=H_{i}$ for $i=1, \ldots, d$. Moreover, $B_{\underline{n}}$ is the tensor algebra of $H_{\underline{n}}$ over $L_{\underline{n}}$. The sheaf $T_{\underline{n}}:=\bar{\oplus}_{i=0}^{d} \mathcal{O}_{X}\left(-n_{i}\right)$ is a left $B_{\underline{n}^{-}}$ module: its decomposition gives it the structure of a left $L_{\underline{\underline{n}}}$-module, and the evaluation map ev: $H_{\underline{n}} \otimes_{L_{\underline{n}}} T_{\underline{n}} \rightarrow T_{\underline{n}}$ describes the $H_{\underline{\underline{n}}}$-action. Since

$$
\operatorname{End}_{\mathcal{O}_{X}}\left(T_{\underline{n}}\right)=\bigoplus_{i \leqslant j} H^{0}\left(\mathcal{O}_{X}\left(n_{j}-n_{i}\right)\right),
$$

the evaluation maps determine a map $B_{\underline{n}} \rightarrow \operatorname{End}_{\mathcal{O}_{X}}\left(T_{\underline{n}}\right)$ that is surjective if $H^{0}\left(\mathcal{O}_{X}\right)=k$. Let $A_{\underline{n}}$ be the path algebra of $\left(K_{\underline{n}}, \mathcal{R}_{\underline{n}}\right)$. Then $T_{\underline{n}}$ is also a left $A_{\underline{\underline{n}}}$-module and $A_{\underline{n}}=\operatorname{End}_{\mathcal{O}_{X}}\left(T_{\underline{n}}\right)$, if $H^{0}\left(\mathcal{O}_{X}\right)=k$. Moreover, the functor

$$
\Phi_{\underline{n}}:=\operatorname{Hom}\left(T_{\underline{n}},-\right): \operatorname{Coh}(X) \rightarrow \operatorname{Rep}\left(K_{\underline{n}}, \mathcal{R}_{\underline{n}}\right) \cong \operatorname{Mod}-A_{\underline{n}}
$$

has left adjoint $\Phi_{\underline{n}}^{\vee}=-\otimes_{A_{\underline{n}}} T_{\underline{n}}$.

We want to find a stability parameter $\theta=\theta_{\underline{n}}(P) \in \mathbb{Z}^{d+1}$ for representations of $K_{\underline{n}}$ of dimension vector $d_{\underline{n}}(P)$ such that $\Phi_{\underline{n}}$ sends semistable sheaves to $\theta$-semistable quiver representations for $\underline{n} \gg 0$ (that is, for $n_{d} \gg n_{d-1} \gg \cdots \gg n_{0} \gg 0$ ). Let

$$
\theta_{\underline{n}}(P):=\left(\theta_{0}, \ldots, \theta_{d}\right), \quad \text { where } \theta_{i}:=\sum_{j<i} P\left(n_{j}\right)-\sum_{j>i} P\left(n_{j}\right) ;
$$

then $\sum_{i=0}^{d} \theta_{i} P\left(n_{i}\right)=0$. The following lemma demonstrates that this is a suitable choice.

Lemma 5.11. Let $\theta=\theta_{\underline{n}}(P)$ be as above and $\mathcal{E}$ be a sheaf on $X$. If, for all $j<i$, we have

$$
\frac{h^{0}\left(\mathcal{E}\left(n_{j}\right)\right)}{h^{0}\left(\mathcal{E}\left(n_{i}\right)\right)} \leqslant \frac{P\left(n_{j}\right)}{P\left(n_{i}\right)},
$$

then $\theta\left(W_{\mathcal{E}}\right) \geqslant 0$.

Proof. By definition, $\theta\left(W_{\mathcal{E}}\right):=\sum_{i=0}^{d} \theta_{i} h^{0}\left(\mathcal{E}\left(n_{i}\right)\right)$, and so it follows that

$$
\theta\left(W_{\mathcal{E}}\right)=\sum_{i}\left(\sum_{j<i} P\left(n_{j}\right)-\sum_{j>i} P\left(n_{j}\right)\right) h^{0}\left(\mathcal{E}\left(n_{i}\right)\right)=\sum_{i} \sum_{j<i} P\left(n_{j}\right) h^{0}\left(\mathcal{E}\left(n_{i}\right)\right)-P\left(n_{i}\right) h^{0}\left(\mathcal{E}\left(n_{j}\right) \geqslant 0\right.
$$

by using the given inequalities.

Corollary 5.12. Let $P$ be a Hilbert polynomial; then for $\underline{n} \gg 0$, we have

$$
\Phi_{\underline{n}}\left(\mathcal{C o h}_{P}^{S S}(X)\right) \subset \mathcal{R}_{e p}^{\theta_{\underline{\underline{n}}}(P)}\left(K_{\underline{n}}\right) .
$$

Finally, we need to pick a suitable stability parameter $\alpha \in \mathbb{N}^{d+1}$. The following lemma, whose proof is analogous to that of Lemma 5.11, shows that we should pick

$$
\alpha=\alpha_{\underline{n}}(P):=\left(\alpha_{0}, \ldots, \alpha_{d}\right), \quad \text { where } \alpha_{i}:=\sum_{j<i} P\left(n_{j}\right)+\sum_{j>i} P\left(n_{j}\right) .
$$

Lemma 5.13. Let $\theta=\theta_{\underline{n}}(P)$ and $\alpha=\alpha_{\underline{n}}(P)$ be as above and $\mathcal{E}$ and $\mathcal{F}$ be two sheaves on $X$. If, for all $j<i$, we have

then

$$
\frac{h^{0}\left(\mathcal{E}\left(n_{j}\right)\right)}{h^{0}\left(\mathcal{E}\left(n_{i}\right)\right)} \leqslant \frac{h^{0}\left(\mathcal{F}\left(n_{j}\right)\right)}{h^{0}\left(\mathcal{F}\left(n_{i}\right)\right)}
$$

$$
\frac{\theta\left(W_{\mathcal{E}}\right)}{\alpha\left(W_{\mathcal{E}}\right)} \geqslant \frac{\theta\left(W_{\mathcal{F}}\right)}{\alpha\left(W_{\mathcal{F}}\right)}
$$




\section{STRATIFICATIONS IN MODULI PROBLEMS}

Definition 5.14. For $\tau=\left(P_{1}, \ldots, P_{s}\right) \in \operatorname{HNT}(X, P)$ and a tuple $\underline{n}=\left(n_{0}, \ldots, n_{d}\right)$, we let

$$
\gamma_{\underline{n}}(\tau):=\left(d_{\underline{n}}\left(P_{1}\right), \ldots, d_{\underline{n}}\left(P_{s}\right)\right) .
$$

Then, for $\tau$ as above and for $\underline{n} \gg 0$ (depending on $\tau$ ), the tuple $\gamma_{\underline{n}}(\tau)$ of dimension vectors of representations of $K_{\underline{n}}$ is a HN type with respect to $\left(\theta_{\underline{n}}(P), \alpha_{\underline{n}}(P)\right)$.

Theorem 5.15. For a $H N$ type $\tau=\left(P_{1}, \ldots, P_{s}\right)$ of sheaves on $X$ with Hilbert polynomial $P$, we have, for $\underline{n} \gg 0$, that

$$
\Phi_{\underline{n}}\left(\mathcal{C o h}_{P}^{\tau}(X)\right) \subset \mathcal{R}_{\operatorname{ep}_{d_{\underline{n}}}(P)}^{\gamma_{\underline{n}}(\tau)}\left(K_{\underline{n}}\right),
$$

where the HN stratification of $\mathcal{R} \operatorname{ep}\left(K_{\underline{n}}\right)$ is taken with respect to $\left(\theta_{\underline{n}}(P), \alpha_{\underline{n}}(P)\right)$.

Proof. To choose $\underline{n}$, we first take $n_{0}$ sufficiently large that all semistable sheaves with Hilbert polynomial $P_{i}$ are $n_{0}$-regular. We choose $n_{1}, \ldots, n_{d}$ successively such that for $0 \leqslant j<l \leqslant d$, we have (1) for $i=1, \ldots, s$, Theorem 5.1 holds for $P_{i}$ and $(n, m)=\left(n_{j}, n_{l}\right)$ and $(2)$ for $i=1, \ldots, s-1$, we have

$$
\frac{P_{i}\left(n_{j}\right)}{P_{i}\left(n_{k}\right)}>\frac{P_{i+1}\left(n_{j}\right)}{P_{i+1}\left(n_{l}\right)}
$$

The proof is almost identical to that of Theorem 5.7 but uses Lemma 5.13. The only part that differs, retaining the notation of Theorem 5.7, is the proof that $W_{i} \cong \Phi_{\underline{n}}\left(\mathcal{E}_{i}\right)$ is $\left(\theta_{\underline{n}}(P), \alpha_{\underline{n}}(P)\right)$ semistable. For any subrepresentation $W^{\prime} \subset W_{i}$, we must check that

$$
\theta_{\underline{n}}(P)\left(W^{\prime}\right) \alpha_{\underline{n}}(P)\left(W_{i}\right)-\theta_{\underline{n}}(P)\left(W_{i}\right) \alpha_{\underline{n}}(P)\left(W^{\prime}\right) \geqslant 0 .
$$

To establish this inequality, we use the fact that $\mathcal{E}_{i}$ is $n_{0}$-regular and semistable with Hilbert polynomial $P_{i}$. Given our choice of $\underline{n}$, we know, for $j<l$, that $\Phi_{n_{j}, n_{l}}\left(\mathcal{E}_{i}\right)$ is $\theta_{n_{j}, n_{l}}\left(P_{i}\right)$-semistable by Theorem 5.1. Hence, $\theta_{n_{j}, n_{l}}\left(P_{i}\right)\left(W_{j, l}^{\prime}\right) \geqslant 0$ for any subrepresentation $W_{j, l}^{\prime} \subset \Phi_{n_{j}, n_{l}}\left(\mathcal{E}_{i}\right)$. Finally to verify (5.5), it suffices to consider the subrepresentations $W_{j, l}^{\prime}$ induced by $W^{\prime}$.

Remark 5.16. To determine a polynomial $P(x)$ of degree at most $d$, it suffices to know the value $P$ takes at $d+1$ different values of $x$. Therefore, for $d \geqslant \operatorname{dim} X$, the map $P \mapsto d_{\underline{n}}(P)$ is injective for all tuples $\underline{n}$ of strictly increasing natural numbers. It follows that the mapping of HN types $\tau \mapsto \gamma_{\underline{n}}(\tau)$ is also injective for $d \geqslant \operatorname{dim} X$. For $d<\operatorname{dim} X$, the mapping on HN types is not injective (cf. Remark 5.8 and Lemma 4.22), and so two HN strata for sheaves can be sent to the same quiver HN stratum. If we take $d \geqslant \operatorname{dim} X$, then this does not happen and, moreover, the above theorem could be used to investigate whether there exists a sheaf of a given HN type.

\section{ACKNOWLEDGEMENTS}

The author is very grateful to the referees for many helpful comments and interesting questions, which greatly improved the paper.

\section{REFERENCES}

AB83 M.F. Atiyah and R. Bott, The Yang-Mills equations over Riemann surfaces, Philos. Trans. Roy. Soc. London Ser. A 308 (1983), no. 1505, 523-615; https://doi.org/10.1098/rsta. 1983.0017.

AK07 L. Álvarez-Cónsul and A. D. King, A functorial construction of moduli of sheaves, Invent. Math. 168 (2007), no. 3, 613-666; https://doi.org/10.1007/s00222-007-0042-5. 


\section{Hoskins}

BFK17 M. Ballard, D. Favero and L. Katzarkov, Variation of geometric invariant theory quotients and derived categories, J. reine angew. Math., published online on 13 February 2016, https://doi. org/10.1515/crelle-2015-0096, to appear in print.

BGL94 E. Bifet, F. Ghione and M. Letizia, On the Abel-Jacobi map for divisors of higher rank on a curve, Math. Ann. 299 (1994), no. 4, 641-672; https://doi.org/10.1007/BF01459804.

Das92 G. D. Daskalopoulos, The topology of the space of stable bundles on a compact Riemann surface, J. Differential Geom. 36 (1992), no. 3, 699-746; https://doi.org/10.4310/jdg/1214453186.

DH98 I. V. Dolgachev and Y. Hu, Variation of geometric invariant theory quotients, Publ. Math. Inst. Hautes Etudes Sci. 87 (1998), no. 1, 5-51; https://doi.org/10.1007/BF02698859.

GD60 A. Grothendieck and J. Dieudonné, Éléments de géométrie algébrique. I. Le langage des schémas, Publ. Math. Inst. Hautes Études Sci. 4 (1960), 5-228; http://www.numdam.org/articles/ PMIHES_1960_-_4_-5_0.

GD63_ Éléments de géométrie algébrique. III. Étude cohomologique des faisceaux cohérents. Seconde partie, Publ. Math. Inst. Hautes Études Sci. 17 (1963), 5-91; http://www.numdam. org/articles/PMIHES_1963_-17__5_0.

GSZ15 T.L. Gómez, I. Sols and A. Zamora, A GIT interpretation of the Harder-Narasimhan filtration, Rev. Mat. Complut. 28 (2015), no. 1, 169-190; https://doi.org/10.1007/ s13163-014-0149-3.

Hes79 W. H. Hesselink, Desingularizations of varieties of nullforms, Invent. Math. 55 (1979), no. 2, 141-163; https://doi.org/10.1007/BF01390087.

HK12 V. Hoskins and F. Kirwan, Quotients of unstable subvarieties and moduli spaces of sheaves of fixed Harder-Narasimhan type, Proc. Lond. Math. Soc. 105 (2012), no. 4, 852-890; https: //doi.org/10.1112/plms/pds022.

Hal14 D. Halpern-Leistner, On the structure of instability in moduli theory, 2014, arXiv:1411.0627.

Hal15_ The derived category of a GIT quotient, J. Amer. Math. Soc. 28 (2015), no. 3, 871-912; https://doi.org/10.1090/S0894-0347-2014-00815-8.

HL97 D. Huybrechts and M. Lehn, The geometry of moduli spaces of sheaves, Aspects Math., vol. E31 (Friedr. Vieweg \& Sohn, Braunschweig, 1997).

HN75 G. Harder and M.S. Narasimhan, On the cohomology groups of moduli spaces of vector bundles on curves, Math. Ann. 212 (1975), no. 3, 215-248; https://doi.org/10.1007/BF01357141.

Hos14 V. Hoskins, Stratifications associated to reductive group actions on affine spaces, Q. J. Math. 65 (2014), no. 3, 1011-1047; https://doi.org/10.1093/qmath/hat046.

Kem78 G. R. Kempf, Instability in invariant theory, Annof Math. 108 (1978), no. 2, 299-316; https: //doi.org/10.2307/1971168.

Kin94 A.D. King, Moduli of representations of finite-dimensional algebras, Quart. J. Math. Oxford Ser. (2) 45 (1994), no. 180, 515-530; https://doi.org/10.1093/qmath/45.4.515.

Kir84 F. C. Kirwan, Cohomology of quotients in symplectic and algebraic geometry, Math. Notes, vol. 31 (Princeton Univ. Press, Princeton, NJ, 1984); https://doi.org/10.1007/BF01145470.

LM00 G. Laumon and L. Moret-Bailly, Champs algébriques, Ergeb. Math. Grenzgeb. (3), vol. 39 (Springer-Verlag, Berlin, 2000).

MFK94 D. Mumford, J. Fogarty and F. Kirwan, Geometric invariant theory, 3rd ed., Ergeb. Math. Grenzgeb. (2), vol. 34 (Springer-Verlag, Berlin, 1994); https://doi.org/10.1007/ 978-3-642-57916-5.

Mum66 D. Mumford, Lectures on curves on an algebraic surface, Ann. of Math. Stud., vol. 59 (Princeton Univ. Press, Princeton, N.J., 1966).

Nes84 L. Ness, A stratification of the null cone via the moment map (with an appendix by David Mumford), Amer. J. Math. 106 (1984), no. 6, 1281-1329; https://doi.org/10.2307/2374395.

Nit11 N. Nitsure, Schematic Harder-Narasimhan stratification, Internat. J. Math. 22 (2011), no. 10, 1365-1373; https://doi.org/10.1142/S0129167X11007264. 


\section{STRATIFICATIONS IN MODULI PROBLEMS}

Rei03 M. Reineke, The Harder-Narasimhan system in quantum groups and cohomology of quiver moduli, Invent. Math. 152 (2003), no. 2, 349-368; https://doi.org/10.1007/s00222-002-0273-4.

Rud97 A. Rudakov, Stability for an abelian category, J. Algebra 197 (1997), no. 1, 231-245, https: //doi.org/10.1006/jabr.1997.7093.

Sha77 S. S. Shatz, The decomposition and specialization of algebraic families of vector bundles, Compos. Math. 35 (1977), no. 2, 163-187; http://www.numdam.org/item?id=CM_1977__35_2_163_0.

Sim94 C. T. Simpson, Moduli of representations of the fundamental group of a smooth projective variety. I, Publ. Math. Inst. Hautes Études Sci. 79 (1994), no. 1, 47-129; https://doi.org/10. $1007 / \mathrm{BF} 02698887$.

Tha96 M. Thaddeus, Geometric invariant theory and flips, J. Amer. Math. Soc. 9 (1996), no. 3, 691-723; https://doi .org/10.1090/S0894-0347-96-00204-4.

Zam14 A. Zamora, On the Harder-Narasimhan filtration for finite dimensional representations of quivers, Geom. Dedicata 170 (2014), 185-194; https://doi.org/10.1007/s10711-013-9876-8.

Victoria Hoskins hoskins@math.fu-berlin.de

Freie Universität Berlin, Arnimallee 3, 14195 Berlin, Germany 